\title{
XI.
}

Königl. chirurgisches Institut in Rom unter der Direction des Professors Francesco Durante.

\section{Ueber die Reparationsprocesse der Schädelknochen mit Bezug auf die gewöhnlich angewandten Methoden der Cranioresection.}

(Experimenteller und pathologisch-anatomischer Beitrag zum Studium der Schädellappen.)

$$
\begin{gathered}
\text { Von } \\
\text { Dr. Nello Biagi, } \\
\text { Assistent. }
\end{gathered}
$$

(Mit 11 Abbildungen.)

Mit dem Studium der Reparationsprocesse der Schädelknochen verbinden sich sowohl wissenschaftliche als praktische Fragen vom höchsten Interesse: es handelt sich in der That darum, zu constatiren, welche von den gewöhnlich angewandten Methoden der Schädelresection am besten dem Zweck entspricht oder geringere Uebelstände mit sich bringt und genau festzustellen, welche Rolle in diesen Processen den einzelnen constitutiven Elementen des Schädeldaches (Knochen, Periost, Dura) zukommt und ob ein Knochenstück, das von seinem Mutterboden losgelöst und reimplantirt ist, noch im Stande ist, im wahren Sinne des Wortes seine Lebensfähigkeit zu bewahren (wie einige Autoren angenommen haben) und zu sehen, wie ein solcher Einheilungsprocess zu erklären ist.

Die Processe der Schädelresection sind, in Ansebung der mannigfaltigen Technik, unzählige; jedoch lassen sie sich mit Fug und Recht in vier Kategorien bringen, je nachdem man eine definitive Resection vornimmt, ohne das resecirte Knochenstück zu reimplantiren, oder eine temporäre, mit Reimplantation der resecirten und von den weichen Theilen völlig abgetrennten Discus, oder indem man einen osteoplastischen Process ausführt, der die temporäre Resection des Knochens 
in seiner ganzen Dicke, aber anhangend an den weichen Theilen zur Grundlage hat; oder indem man, nach dem osteoplastischen Process Durante's, kleine Knochenbruchstücke am Periost hängen lässt.

Durch ein genaues analytisches und systematisches Studium, das den Verlauf des Reparationsprocesses in jedem Fall verfolgt und dureb die scharfe Beobachtung des Verhaltens der verschiedenen Coëfficienten der Wiederherstellung, können wir in Stand gesetzt werden, über die Vorzüge und Mängel jeder Methode mit Sicherheit zu urtheilen und uns darüber zu entscheiden, welche von diesen Methoden wir heutigen Tages vorzuziehen haben. In diesem Urtheil wollen wir uns nicht von vagen, mehr oder weniger begründeten theoretischen Anschauungen, sondern lediglich von dem Grundsatze Broca's leiten lassen, wonach diejenige Methode als die beste zu betrachten ist, die auf's einfachste und schnellste dem Schädel seine natürliche Widerstandsfähigkeit zurückzugeben im Stande ist.

Mit dieser Frage, die vom höchsten praktischen Interesse ist, hängen andere zusammen, die gleichfalls eine grosse Bedeutung für die Histogenesis und für die Pathologie des Knochengewebes besitzen; ich deute auf das osteogenetische Vermögen der Dura und des Periosts hin und auf das Schicksal des von den weichen Theilen befreiten und wieder in den Defect implantirten oder am Periost ganz oder in kleinen Bruchstücken hängen gebliebenen Knochens.

Die meisten Autoren stimmen darin überein, dass sie der Dura und dem Periost ein osteogenetisches Vermögen bei der Wiederherstellung der Schädelknochen zuschreiben; die Meinungen gehen jedoch auseinander, sobald jeder einzelne Forseher den Grad dieses Vermögens festzustellen sucht. Die einen, zuerst Ollier, tragen, obwohl sie das knochenbildende Vermögen der Dura annehmen, kein Bedenken, dem Periost die Hauptrolle zuzuertheilen, während andere der Dura eine vorwiegende Wirkung beilegen, wie Klencke, Cornil, Flourens und Berezowsky; ja Berezowsky gelangt in seiner jüngsten Schrift zu dem experimentellen Schluss, dass die Wiederherstellung des Schädeldefects nur möglich ist, wenn die Dura unverletzt bleibt. Zu diesem Zweck durchbohrt er einen Kaninchenschädel an beiden Seiten mit einem Trepan von $6 \mathrm{~mm}$ Durchmesser; in einem der beiden Defecte nimmt er die Dura fort, während er dieselbe in dem anderen verbleiben lässt und schneidet das Periost im Umfang der Trepankrone aus. Indem er die Thiere zu verschiedenen Zeiten tödtete, stellte er fest, dass die Knochenbildung nur dort, wo die Dura unverletzt ist, stattfindet und in diesem Fall in üppiger Weise vor sich geht; dieselbe beginnt am 5. Tage und am 7. ist bereits Knochen- 
gewebe im Defect vorhanden, bis zwischen dem 25. und 30. Tage die Wiederherstellung vollkommen ist. Pascale leugnet zwar die Mitwirk ung des Periosts nicht ab, nimmt jedoch an, dass dieses erst später und mit geringerer Intensivität als die Dura und der Knochen seinen Einfluss ausübt. Nach Vignolo und Ca miniti dagegen hat die Dura eine weit beschränktere Bedeutung im Reparationsprocess des Schädels. Heine und Pontet endlich legen dem Knochen ausschliessliche Bedeutung bei, dem Periost und der Dura gar keine.

Die Ansichten gehen jedoch viel weiter auseinander, sobald es sich darum handelt, festzustellen, welches Schicksal dem von den weichen Theilen befreiten und in den Defect reimplantirten Knochenstiick zufällt: ist dieses im Stande, sein Leben fortzusetzen, oder geht es in Nekrose über? Und was wird aus demselben, wenn man es an den weichen, nicht völlig von den umgebenden Geweben getrennten, aber mit diesen an der schmalsten Stelle verbundenen Theilen belässt?

Hier kommt natürlich die ganze wissenschaftliche Auffassung in Bezug auf Knocheninnest überhaupt mit ihrer endlosen Literatur und den disparatesten Schlussfolgerungen in's Spiel, und zwar ebensowohl auf dem experimentellen, als auf dem klinischen Gebiet. So berichtet Chipault von 73 Fällen von mit Erfolg operirten Individuen, die einer Schädelresection mit Autoplastik unterworfen wurden; man darf jedoch nicht verschweigen, dass viele, ja die grösste Anzahl dieser Fälle zu jung sind, um ein genaues statistisches Urtheil zu erlauben: nur in den Fällen von W ed emeyer ( 7 Jahre), M a c Ew en, (22 Jahre), Gerster (einige Monate) und wenigen Anderen kann man zuversichtlich behaupten, dass es sich um einen guten und andauernden Ausgang handelt. Ollier dagegen behauptet zwar, dass der herausgeschnittene Knochen seine Lebenskraft behalten kann und führt zum Beweis die Resultate von Autopsien bei vor langer Zeit operirten Individuen an; warnt aber in seinem letzten Buch (De l'osteogénèse chirurgicale) die Operateure vor dieser Methode, welche in der Praxis ernste Folgen nach sich ziehen könne, indem sie im Verlauf Compression, Irritation, Suppuration und in Folge von dieser Ausfallen des Knochens selbst hervorzurufen im Stande wäre, und fuhrt zur Stiitze seiner Ansicht experimentelle und klinische Fälle an. Dass der französsische Chirurg Recht hat, beweist der Fall von Jaboulay, ferner die beiden Fälle von $\mathrm{Y}$ ones und der von Franck und Churk, in denen Eiterung eingetreten ist. - Jedoch muss ich sagen, dass die Kliniker, die sich mit diesem Gegenstande beschäftigt haben, fast ausschliesslich den groben makroskopischen Befund in 
Betracht zogen; ihre Behauptungen können daher kein grosses Gewicht für uns besitzen. Auch haben die betreffenden experimentellen Studien bis jetzt zu keinem sicheren Ergebniss geführt. Ollier, Wolff, Buscarlet, Schmitt und Laurent setzten die Lebenskraft des Knochenstïckes nicht in Zweifel, und bis zum Jahre 1893 konnte die Frage als gelöst angesehen werden, als Barth in zwei auf einander folgenden Mittheilungen an den deutschen Chirurgencongress mit Einwendungen hervortrat, denen er in seiner klassischen Schrift von 1895 eine concrete Gestalt gab. Hier leugnete er absolut die Lebenskraft dieser reimplantirten Knochenstücke. - So wurde die Discussion auf's Neue eröffnet und David (1896) spricht weder von Nekrose, noch von wahrer und eigentlicher Heilung, sondern von einer besonderen Art von Lebenbleiben, das sich in einem Herabsinken der Lebenskraft in den Knochenelementen ausspricht, einer transitorischen Verminderung, die den Lebergang zur völligen Restitutio ad integrum bildet. - Auch in Italien ist die Frage lebhaft besprochen worden und in der letzten Zeit sind einige vortreffliche Arbeiten über diesen Gegenstand erschienen; ich erwähne die Schrift Sacchi's, der beobachtet hat, dass die aus dem Schädel herausgeschnittenen und reimplantirten Knochenstücke gewöhnlich einem centralen Schwund ausgesetzt sind, der mit einer vollständigen Zerstörung des Knochens endigt, an dessen Stelle ein faseriges Bindegewebe tritt. Er fand, dass nur ausnahmsweise die Lebenskraft solcher Stücke erhalten geblieben war. Auch der Abbandlung Pascule's muss ich gedenken; nach der Ansicht dieses Forschers verfällt der reimplantirte Knochen der Regel nach der Nekrose, jedoch ausnahmsweise bleiben einige Gruppen cellulärer Elemente am Leben, namentlich bei jungen Thieren von hohem, osteogenetischem Vermögen. Die Absorption und die Ersetzung des nekrotischen Stuckes gehen nach ihm gleichzeitig vor sich. Die Substitution wird dadurch charakterisirt, dass sie, statt progressiv zu verlaufen, in einem gewissen Moment, gewöhnlich sehr früh, stehen bleibt und im Knochen ein Schwundprocess stattfindet, in Folge dessen die Ueberbleibsel des reimplantirten Stückes noch nach langer Zeit sichtbar sind, was mit Barth's Ansicht im Widerspruch steht. Valan endlich nimmt an, dass die Knochenscheibe einer nur theilweisen Nekrose verfällt und verlegt die am Leben erhaltenen Theile an die Peripherie, wäbrend der centrale Theil der Nekrose ausgesetzt ist, aufgezehrt wird und schliesslich durch die osteogenetische Thätigkeit des Granulationsgewebes, das den implantirten Knochen umgiebt und durchzieht, ersetzt wird. Kürzlich hat Valerio die Frage wieder aufge- 
nommen; er unterscheidet Knochenstïcke, die den Defect vollständig und hermetisch verschliessen und solche, die zwischen sich und den Ründern des Defectes einen gewissen Raum frei lassen; er kommt hiernach zu dem Schluss, dass die ersteren die Lebenskraft bewahren, während die letzteren absterben und verschwinden.

Dagegen findet ein ganz anderes Verhalten statt bei einem Knochenstück, welches ausgeschnitten ist, jedoch mit den weichen Theilen in Zusammenhang bleibt; in diesem Fall bildet das Leben die Regel und die hicrauf bezüglichen Versuche Pascale's haben die Schlüsse Ollier's nur bestätigen können. An diesem Punkt steht die Frage im gegenwärtigen Augenblick; es handelt sich, wie ich wiederhole, um Fragen, die nicht nur theoretischen und doctrinellen Werth haben, sondern von deren präciser Lösung die definitive Wahl einer osteoplastischen Metbode abhängt, die den vorher von uns aufgestellten Bedingungen, nämlich der Einfachheit, der schnellen Ausführung, Resistenz und Sicherheit des Erfolges vollkommen entspricht.

\section{Die osteoplastische Methode Durante's.}

Einige haben die Priorität dieser Methode Müller zuschreiben wollen, der in einer 1890 im Centralblatt für Chirurgie veröffentlichten Mittheilung vorschlug, die äussere Hüllenschicht dem lappen anhängen zu lassen.

Vor allen Dingen bemerke ich, dass Durante zum ersten Male im Mai 1884 eine Operation mit seinem Lappen ausgeführt hat, d. h. 5 Jahre, bevor die Abhandlung Müller's erschienen ist; ausserdem muss ich in technischer Beziehung hervorheben, dass die beiden Processe nichts mit einander gemein haben.

Müller schlägt in der That geradezu die Ausschneidung der ganzen äusseren Hüllenschicht vor, während Duran te sich darauf beschränkt, Knochenbruchstï̈cke am Periost anhängen zu lassen. Wenn nun Durante's Methode für den in der chirurgischen Praxis Erfahrenen leicht auszuführen ist, so muss diejenige Müller's als eine solche bezeichnet werden, deren Durchführbarkeit wenigstens problematisch ist; und dies ist nicht nur etwa meine Ansicht, sondern auch die Broca's und Maubrac's, welche diese Methode kritisiren. Auch die Anschauungen, die den Ausgangspunkt beider Verfahren bilden, sind durchaus verschieden; in der That geht $\mathrm{M}$ u $\mathrm{l}$ e $\mathrm{r}$ darauf aus, dass der Knochen seine Lebenskraft bewahre, während $D$ urante nur auf das Reizungsvermögen desselben und seine Function als einfaches Modell für die zukünftige Knochenneubildung Gewicht legt, so dass es sich schliesslich nur um passive, zur Absorption bestimmte Modelle handelt. 
Mit König's Verfahren besteht nur eine entfernte Verwandtschaft, da dieser Autor an jeder der beiden den Defect bildenden Klappen nur ein Fragment anhängen lässt, welehes er abschneidet, indem er die Knochenhöhlung mit einem grossen Meissel ausglättet. Dies Fragment ist nothwendig zu klein im Verhältniss zu dem Defect. Nach Voraufschickung dieser wenigen Thatsachen, die ich feststellen wollte, schreite ich zur Beschreibungr der Technik.

Die Section der weichen Theile wird entweder halbkreisförmig mit der Basis unten ausgeführt oder vermittelst eines längeren Hauptschnittes und zweier kürzerer perpendiculärer, die mit ihrem Mittelpunkt auf den Enden des ersteren stehen, so dass zwei rechtwinkelige Klappen gebildet werden. Diese Schnitte dringen allenthaben bis auf den Knochen. Nachdem der I appen so vorgezeichnet und für die Blutstillung gesorgt ist, wird der Knochen vermittelst eines Raspatoriums bis zu einem gewissen Grade von den ihn umgebenden weichen Theilen befreit und darauf mit einem feinen ILohlmeissel die Linie der Knochensection gezogen, parallel mit dem Rande der weichen Theile. Nach Vollendung dieses ersten Operationsactes befreit man mit einem gewöhnlichen Ma c Ewen - Meissel einen kleinen am Periost anhängenden Knochensplitter der äusseren IIüllenschicht. Es ist von Bedeutung, den Meissel so zu halten, dass er soweit als möglich die Richtung einer Tangente zur Knochenoberfläche einnimmt, indem man so oberflächlich als möglich kleine Schläge ausführt und zugleich den Meissel nach jedem Schlage leise aufhebt. So erhält man einen Iappen, so gross man will, der sehr beweglich und leicht verschiebbar ist. Ist man an diesem Punkt angelangt, so handelt es sich nur darum, den Schädeldefect so weit und gross wie den Lappen der weichen Theile zu machen, was entweder auf einmal mit dem Hohlmeissel oder mit der Zange von Montenovesi ausgeführt wird.

Dieses Verfahren habe ich in vielen Fällen von Durante ausführen sehen. Vier von diesen Fällen bin ich in der Lage gewesen, auf's Neue wieder zu sehen, zwei in einer ambulatorischen Klinik, die anderen beiden am Operationstisch, da es sich um Rückfälle handelte. In allen Fällen war der Knochen vollkommen wiederhergestellt. Das Aiter des Lappens belief sich bei den beiden auf's Neue Operirten in einem Fall auf 8 , im anderen auf 11 Jahre. In den beiden übrigen Fällen betrug das Alter 2 und 5 Jahre und ich kann versichern, dass vom operativen Gesichtspunkt aus sich keine günstigeren Umstände denken lassen. Von den beiden auf's Neue Operirten habe ich nur von einem das anatomische Stück erhalten und mikroskopisch prüfen können, und zwar das 11 Jahre alte. ... 
Osteoplastischer Lappen Durante's von 11 Jahren. (Frau.)

Die charakteristischen makroskopischen Eigenschaften des erwähnten anatomischen Stückes sind folgende: Dicke schwankend zwischen 1 und $1 \frac{1}{2} \mathrm{~cm}$; zwei feine Schichten compacten Knochens vertreten die äussere Hüllenschicht und die Vitrea, zwischen ihnen befindet sich das schwammige Gewebe; die äussere Hüllenschicht ist natürlich um den ganzen beim zweiten Operationsact am Periost hängen gebliebenen Theil verkleinert, während die Vitrea an ihrer glatten Oberfläche Furchen und Dellen zeigt, die den Gefässen und dem unter ihr befindlichen Neoplasma, um dessen willen die Operation wiederholt wurde, entsprechen.

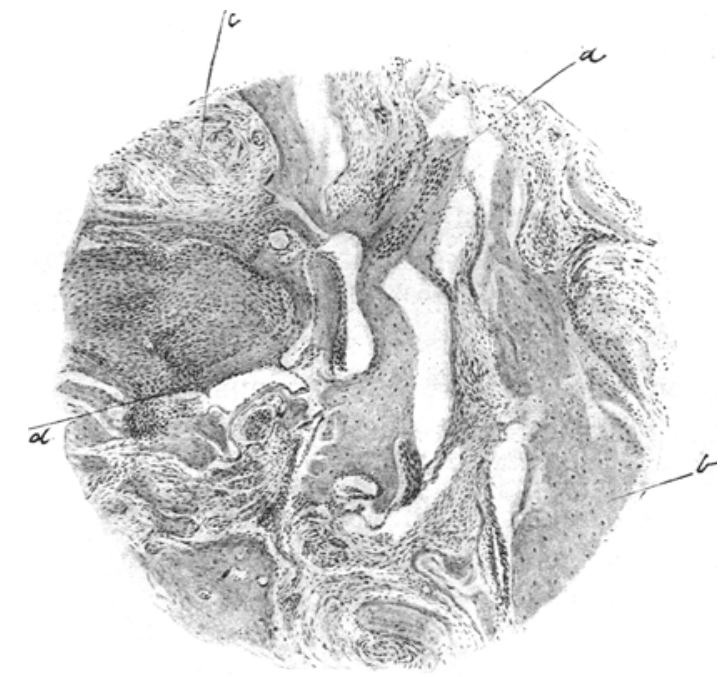

Fig. 1.

Mikroskopischer Befund (Fig. 1). Betrachtet man die verschiedenen aus der Neubildung gemachten Präparate, gefärbt mit doppelter Coloration von Hämatoxylin-Eosin, Hämatoxylin-Orange, Hämatoxylin-Gieson, Anilinblau und Safranin, so bemerkt man:

Bei geringer Vergrösserung (Obj. 4 Kor.) vor Allem die nöthige Wiederherstellung des normalen $\mathrm{Za}$ standes, nämlich der Vitrea wie der äusseren Hullenschicht mit ubereinander gelagerten $\mathrm{Z}_{0}$ nen, so dass wir das Bild einer lamellenartigen Structur vor uns haben, die nur von den Canälen unterbrochen wird, die zum Durchgang der Gefässe bestimmt sind, welche in Längsrichtung durchlaufen und sich bis in die Diploë verfolgen lassen: diese Canäle haben einen verschiedenen Umfang mit ziemlich regelmässigem Lumen. Dieselben zeigen nur in ihrem Verlauf wenig ausgesprochene blasenförmige Erweiterungen, gleichsam varicöse Erscheinungen, die den Windungen des Gefässes in ibrem Innern entsprechen. Das Gefäss ist voll von Blut und fullt den Canal nicht völlig aus, aber zwischen seiner Wand und den Wänden des Canals finden wir zahlreiche grosse spindelförmige Bindegewebselemente mit klar hervortretendem Kern, in concentrischen 'Zonen angeordnct und mit spärlichen Infiltrationselementen vermischt.

Das Aussehen des Knochens ist vollkommen normal in den am meisten peripherischen Schichten; die homogene Grundsubstanz zeigt eine An- 
ordnung in grossen Lamellen, als ob es sich um Uebereinanderlagernng von Knochenschichten landelte, die Zellen mit klar sichtbaren und gut färbbaren Kernen. Hier und dort bemerkt man ziemlich weite haversische Canäle, durch welche die lamellenartige Structur unterbrochen wird, um einer concentrischen Anordnung Platz zu machen.

Wenn wir uns nach und nach von den peripherischen Theilen aus nach den centralen wenden, so beginnen wir in der Grundsubstanz kreisförmige, klarere and mehr homogene Zonen wahrzunehmen, einige grösser, andere kleiner, mit hellerem Kolorit, die in der Section das Aussehen von kreisförmigen, leeren Räumen haben, welche sich bald aneinander legen, bald mit einander verschmelzen, so dass sie ihre regelmässigen Umrisse verlieren und der Grundsubstanz ein köruiges Aussehen verleihen. Dieser Thatsache, die wir als eine atrophische Entartungserscheinung betrachten, entsprechen Canäle, die keine regelmässige Form wie in den am meisten peripherischen Theilen besit\%en, sondern von veränderter Form, vergrössert und reich an Bindegewebselementen sind. In einigen ihrer unregelmässigen Erweiterungen sieht man grosse Flemente mit mehreren Kernen, ähnlich den schon von Kölliker beschriebenen. Bei stärkerer Vergrösserung treten diese Thatsachen noch deutlicher hervor, und wo die Grundsubstanz, wie wir gesagt haben, bei geringer Vergrösserung sich nicht mehr homogen darstellt, sehẹn wir, wie die Kerne bald unregelmässig werden, ihre Form verändern, sich verkleinern, grobkörnig werden und sich stark färben, bald geradezu in Fragmente zerfallen und sich gleichsam pulverisiren, bald klar, homogen hyalinisch erscheinen. Ohne $\mathrm{Zweifel}$ vollzieht sich in diesen tieferen Theilen eine regressive Phase der Aufzehrung des Knochens. Wenn wir uns von der Peripherie entfernen, haben wir nicht mehr die fur diese charakteristische fortlaufende Lamellenstructur; der Typus des Knochens ist durchaus schwammig: in der That bemerkt man eine grosse Anzahl von kleinen Balken, die mit einander verflochten sind und sich in jeder Weise aneinanderlegen und so zur Bildung grosser mit jungem, an Gefässen reichem Bindegewebe angefallter Höhlen Anlass geben. Diese Bälkehen sind bisweilen gross und von ansehnlichem Umfang, an anderen Stellen dagegen haben sie das Aussehen einer sehr feinen Knochenschale, die eine Höhle umschliesst; diese Höhlen stellen sich in der Section bald als unabhängig, vom Krochengewebe völlig umgeben dar; bald dagegen stehen sie untereinander in Zusammenhang und verbinden sich anastomatisch, so dass sie in ein unregelmässiges Balkengerlust ibergehen.

Jas Bindegewebe in den Zwischenräumen zwischen den Balken ist in Strängen und Gehängen spindelförmiger Elemente angeordnet, unter welche Elemente mit einem grösseren, eiförmigen oder runden Kern gemischt sind (offenbar junge Bindegewebselemente) sowie auch in Infiltrationselementen. An einigen Punkten findet $z$ wischen dem Knochen and dem Bindegewebe zwischen den Balken keine scharfe Trennung statt, sondern der Uebergang von einem zum anderen ist allmählich, fast unmerklich. Diese Wabrnehmung gewinnt bei stärkerer Vergrösserung noch bedeutend an Schärfe; man sieht in der That, wie die Grundsubstanz sich aus einer homogenen in eine fein gestreifte verwandelt. Diese Streifen werden im Verlauf immer deutlicher und markirter, indem sie sich im 
Bindegewebe fortsetzen, während die rothe Fuchsinfarbe (Gieson) von dem Knochenbalken nach und nach in das Gelb des Bindegewebes Hbergeht. In den Durchgangszonen sieht man Knochenelemente ihren Kern vergrössern, sich mit einem klaren Hof umgeben und alle charakteristischen Eigenschaften von Bindegewebselementen annehmen. Diese Thatsache offenbart sich ausserdem an einigen Punkten im Innern des Knochenbalkeus selbst, wo die Grundsubstanz nicht mehr roth, sondern gelb gefärbt und gestreift ist, mit völlig vitalen Elementen, die mehr denen des Bindegewebes, als denen des Knochens gleichen und einen gleichfalls grossen, spindelförmigen Kern besitzen. Meiner Meinung nach handelt es sich hier um die Verwandlung des fibrösen Bindegewebes in Knochen und dies wurde in dem Aussehen des Bindegewebes selbst an diesen und anderen ähnlichen Punkten seine Bestätigung finden, sowie in den rothen ,annuances", die in demselben so häufig sind und die Anordnung in Balken ziemlich treu wiedergeben.

Hier und da sieht man an den Knochenbalken angelagert wirkliche Pallisaden vielseitiger, keilförmiger Flemente, die sie bekleiden und unmittelbar in Berübrung mit ihnen die homogene Knochensubstanz, die intensiver und gleichförmiger als der Rest des Balkens gefärbt ist und sich wie eine Binde um den letzteren legt. Bei starker Vergrösserung bemerkt man, dass die oben erwähnten Elemente ein feinkörniges Protoplasma besitzen; der runde, wohl in die Augen fallende Kern ist gegen die dem Balken entgegengesetzte Peripherie verschoben, während der dem letzteren unmittelbar benachbarte Zellenpol von der homogenen Zone der Grundsubstanz umgeben ist und das diesem Pol entsprechende Protoplasma selbst sich roth färbt, zum Unterschied von dem Protoplasma des entgegengesetzten Pols. Bei genauer Beobachtung sieht man in der Grundmasse selbst Kerne, die therall von dieser umgeben sind, während das celluläre Aussehen verloren gegangen ist. Diese Thatsache, die man mit dem Mikroskop Schritt für Schritt verfolgen kann, scheint mir das innere Wesen des Knochenbildungsprocesses klar aufzuhellen und stimmt genau aberein mit dem, was Gegenbaur, Stieda und Waldeyer zu sehen Gelegenheit hatten.

Alles zusammengefast, befinden wir uns einem Knochen in voller Restitutio ad integrum gegenüber mit fort- und rückschreitenden Lebenserscheinungen, und gerade auf die Fortdauer dieser so ausgedehnten progressiven Thatsachen lange nach dem Operationsact möchte ich die Aufmerksamkeit richten, als einen Beweis für die Thätigkeit und den Fortschritt des osteogenetischen Vermögens in den Schädelknochen völlig entwickeiter Individuen.

\section{Experimenteller Theil der Untersuchung des osteoplastischen Lappens Durante's.}

Die Experimente wurden an Hunden angestellt: mit einer der beim Menschen beschriebenen indentischen Technik brachte ich einen grossen Defect in der parietalen Schläfengegend an, um so weit als 
möglich mit den für den Nlenschen geltenden Umständen in Lebereinstimmung zu bleiben. Der Defect batte im Durehschnitt eine Basis von $3-4 \mathrm{~cm}$ auf eine Höhe von 2-21/2 cm, muss also als sehr gross betrachtet werden, wenn man die Dimensionen der Schädel, an denen ich die Versuche anstellte, in Rechnung zieht: immer lag die Basis des Lappens unten. Der einzige Uebelstand, der sich bei dem Experiment herausstellte, bestand in der IIämorrhagie, die jedoch gewöhnlich durch eine leise Compression bald beseitigt wurde; sie hatte ihren Grund in dem weiten Gefässtbum der Diplö̈. Hierauf legrte ieh den Lappen wieder über den Defect und befestigte ilın mit zwei oder drei Nadelstichen am zunïchst gelegenen Theil des Periosts; schliesslich wurden Muskeln und Haut zusammengenäht, um zu vermeiden, dass Fäden der Nadelstiche in der Tiefe blieben. - Die Dura liess ich bei einigen Thieren intact; bei anderen sehnitt ich sie rings an den Rändern des Defects mit einer kleinen Scheere ab, so dass die weichen Meningen sich in unmittelbarer Berührung mit dem Lappen befanden.

Fierzu wurde ich veranlasst, um einen nicht seltenen Fall hervorzurufen, in welchem man den Lappen findet, wenn die Dura wegen $\Lambda$ dhäsionen oder Ausbreitung neoplastischer Processe u. s. w. fortgeschafft werden muss. Auf diese Weise war ich auch in der Lage, das osteogenetische Vermögen der Dura selbst zu beurtheilen.

\section{Osteoplastischer Lappen Durante's; Dura intact.}

Die anatomischen Stücke dieser Reihe wurden 3 per primam geheilten Thieren, die zu verschiedenen Zeiten getödtet waren, entnommen. - An diesen stellte ich meine mikroskopischen Untersuchungen an, indem ich mich, was die Fürbung betrifft, an die schon bei der histologischen Prüfung des menschlichen Stückes erwähnte Technik hielt. Jie anderen Thiere starben am Shock und wurden bei Seite gelassen wegen Suppurationserscheinungen. In Bezug auf diese letzteren muss ich hervorheben, dass ich bei der Autopsie niemals Gehirnbruch zu constatiren hatte; ausserdem waren die Knochenfragmente zum grossen Theil eliminirt und die Dura verdickt, glanzlos und mit einer fibrinösen, eiternden Schicht bedeckt; das makroskopisch normale Gehirn hing kaum an.

18 Tage. Makroskopisch ist die Continuität des Knochens vollkommen: die Dura hängt an, das Gehirn erweist sich seinem Aussehen und seinem anatomischen Charakter nach als normal. Mit einer Stecknadel dringt man durch den Defect, dem entsprechend das durchschnittene Schädoldach ein gleichsam lamellenartiges Ausseluen hat. 
Histologische Prufung. Richten wir unsere Aufmerksamkeit auf den den Defect umgebenden Knochen, so stellt er sich uns in der Section als derselbe Typus eines Fracturstümmels dar, und während derselbe in den weiter abliegenden Theilen vollkommen normal ist, zeigt er in den dem Defect zunächst liegenden Theilen die gewöhnliche nekrotische Binde, die man bei allen Brulchen antrifft und die von Durante so vortrefflich beschrieben worden ist, und schon fruher von Bonome bemerkt war. Diese Binde ist von verschiedener Dicke und setzt sich eine kurze Strecke lang an der Oberfläche der Dura und des Periosts

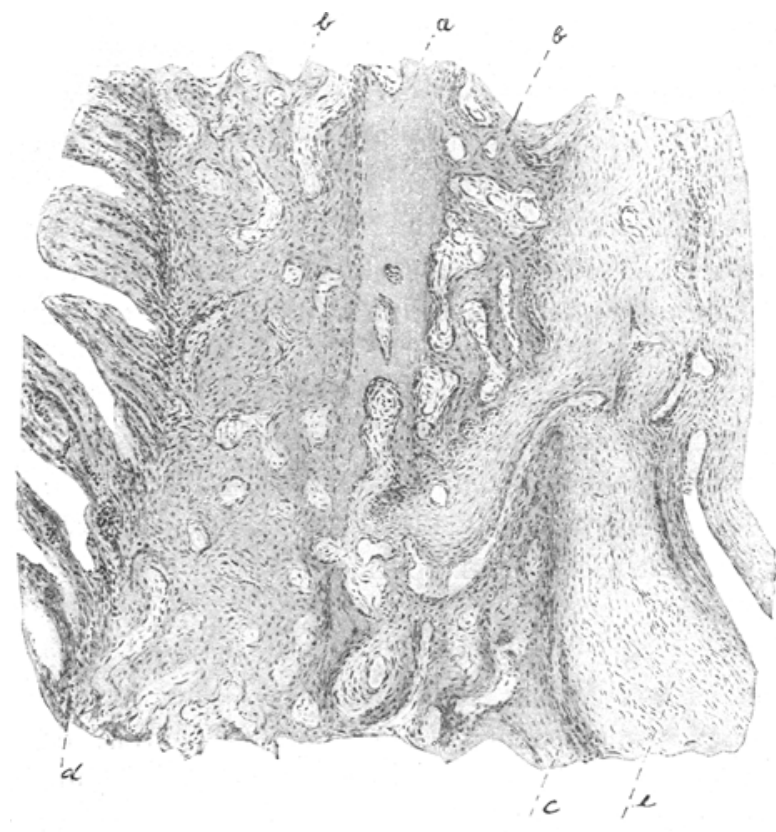

Fig. 2 . fort, uberall von den Elementen dieser Theile angegriffen, die sich dort in Begleitung der Gefässe einnisten und dem Knochen ein unregelmässiges geschlängeltes Aussehen verleihen. Bisweilen handelt es sich um eigentliche Canäle, die in den nekrotischen Theil eindringen und sich mit den haversischen Canälen verbiuden. Diese letzteren haben sich vergrössert und unregelmässig ausgedehnt mit varicösen Erweiterungen, sind reich an Elementen und Gefässen, mit zahlreichen Gigantenzellen; sie durchziehen den Knochen in verschiedenen Richtungen, einige von ibnen öffnen sich am äussersten, den Defect umgebenden Rande. Dann sieht man, wie die Elemente, von denen diese Canäle voll sind, zusammen mit ihren Gefässen sich unter die Elemente des Gewebes mischen, welches den Defect ausfullt und das wir weiterhin beschreiben werden. Periost und Dura befinden sich in den am weitesten abliegenden Theilen in lebhafter Wucherung. Sie haben ihr normales Anssehen verloren und man sieht, dass sie sich in ihren tiefsten Lagen in ein Gewebe verwandelt haben, das sehr reich an Gefässen ist und fast ausschliesslich aus schönen eiförmigen Elementen mit grossem, rundem Kern besteht, die sich in knochenähnlichen Balken anorduen, in denen die interelementare Substanz immer reichlicher wird und schliesslich aus einem feinfaserigen Aussehen in ein hyalinisch homogenes ubergeht, während die Kerne eine pyramidale, 
eiförmige Gestalt annehmen. Schliesslich sieht man sie in wohl umgrenzte Räume eingeschaltet, welche die zukïnftigen Knochenkörperchen darstellen. Bei Coloration mit Anilinblau und Safranin wohnt man der wirklichen Bildung des Knochens in seinen dem frtheren Knochen nächsten Theilen bei, an den sich die erwähnten Balken anschliessen. Zwischen den Balken existiren Höhlungen, die Gefässe und Elemente enthalten; von diesen sind einige echte Infiltrationselemente, andere tragen den Charakter von ursprínglichen duralen und periostalen Elementen und sind ein Wucherungsproduct der Bindegewebselemente. Diese nehmen den Raum zwischen dem Gefäss und der Peripherie des Balkens ein, und am Fnde des letzteren gruppiren sie sich in einer gewissen Ordnung zu einer ununterbrochenen Lage, so dass sie den Balken völlig ausfüllen. Dieselben Thatsachen treten noch viel bestimmter an dem Punkt des Knochendefects hervor, wo der osteoplastische Isappen an die Stelle des Knochens gesetzt ist. Hier verschmilzt sich die Production des Periosts mit der der Dura und beide nehmen eine balkenartige Anordnung an, der dieselben charakteristischen Eigenschaften zukommen, wie der fruber beschriebenen. Diese neugebildeten Balken stitzen sich auf die nekrotischen Knochenlamellen, die aus den durch den Operateur hervorgerufenen Fragmenten bestehen. Dieser Zusammenhang ist durchaus ununterbrochen, und schwerlich würde man die neugebildeten Balken von denen des abgestorbenen Knochens unterscheiden, wenn dazu nicht die verschiedenen Tinctionsfähigkeiten und die anatomischen Charaktereigenschaften verhülfen; denn diese bringen eine ziemlich scharfe Scheidelinie hervor. Die erwähnten Lamellen sind von Canälen mit unregelmässigen, geschlängelten Rändern durchfurcht, die alle von mit Capillargefässen verbundenen Elementen erfullt sind, die sie angreifen und zernagen, wie man aus der Gegenwart von Gigantenzellen entnehmen muss. Einige von diesen Lamellen finden wir geradezu umgeben von einer ununterbrochenen Binde neugebildeten lebenskräftigen Knochens in ubereinander liegenden Streifen. Dieser neugebildete Knochen ruhrt von der Production des Periosts und der Dura her; und dies sind die kleinsten Lamellen, wie man an anderen Punkten sehen kann, wo keine Knochenbildung stattgefunden hat. Man erblickt dann eine Lamelle, zu der die von Osteoblasten umgebenen Gefässschlingen vom Periost und von der Dura aus vorzchreiten, indem sie sich an die Lamelle legen und so rings umher ein osteogenetisches Knochengewebe hervorbringen, das sich bald darauf in Knochen verwandelt. In diesen Präparaten tritt der allmähliche Uebergang des knochenähnlichen Balkens in den Knochenbalken auf's Schärfste hervor. In der That, während im knochenähnlichen Balken die interelementare Substanz ein feinfaseriges Aussehen hat und die Farbe von Bindegewebe annimmt, sieht man sie nach und nach hyalinisch und homogen werden und die unterschiedslose Knochenfarbe annehmen.

2 Monate. Betrachten wir die Präparate an diesem Zeitpunkt, so haben wir höchst interessante Eigenthümlichkeiten an ihnen zu verzeichnen, sowohl in Bezug auf den die Ränder des Defects bildenden Knochen, als an dem Punkt des Defects, wo sich der osteoplastische Lappen befand. Während der arsprüngliche Knochen sich uns in den entferntesten Theilen als völlig normal darstellt, nur mit einer beträchtlichen Erweite- 
rung der haversischen Canäle, bemerken wir dagegen in der Gegend unmittelbar an der Stelle, wo die Continuităt unterbrochen ist, ebensowohl nekrotische, wie völlig vitale Theile. Die ersteren lassen sich an der Gegenwart von missgestalteten verschrumpften Kernen erkennen, die bald eine sehr intensive, bald eine blasse Farbe baben; ferner an leeren oder mit einem grobkörnigen Pulver angefülten Knochenkörperchen. Im Allgemeinen sind dic abgestorbenen Theile von den völlig lebenskräftigen gut zu unterscheiden, wenn sie auch untereinander zusammenhängen und eng aneinander liegen. Die Scheidelinie wird durch eine plötzliche Ver-

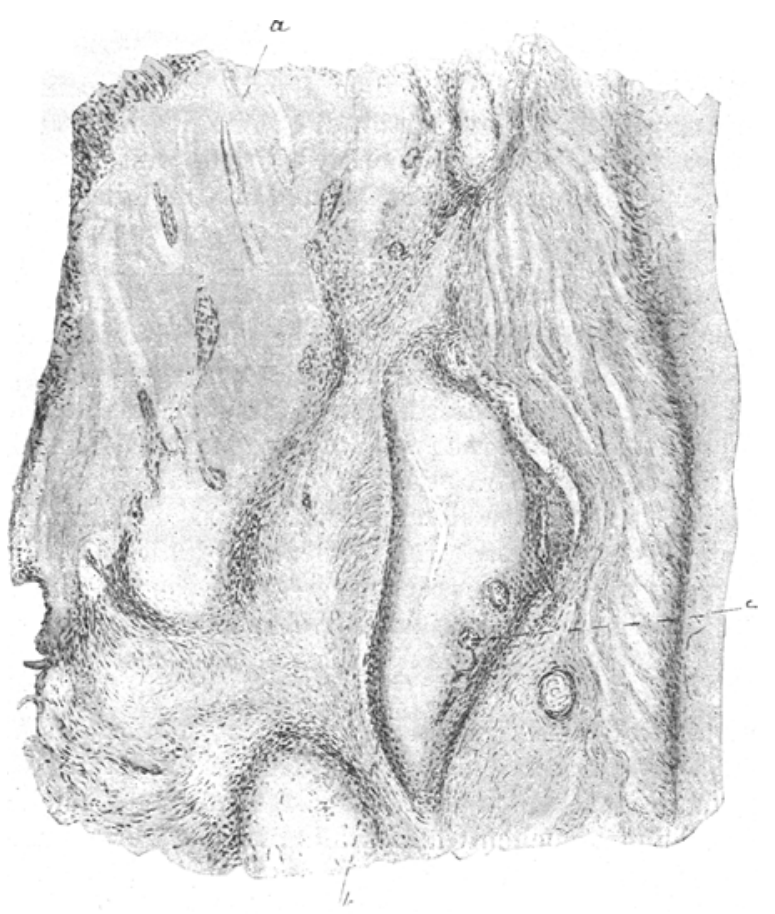

Fig. 3. änderung der Tinctionsfähigkeiten hervorgebracht, in Folge derer die vitalen Theile eine intensivere und $\mathrm{zu}$ gleich gleichförmigere und mehr homogene Färbung annehmen. Es handelt sich um einen ganzen Streifen neagebildeten Knochens, sowohl auf der Seite des Periosts, als auf der der Dura, den man in den Sectionen auf der oberen und unteren Oberfläche der Schädelknochen sieht und der die nekrotischenTheile am Punkt, wo die Unterbrechung desKnochens stattgefunden hat, umhallt and einkap-

selt. Man findet, dass diese nengeformte Knochenschale auf der Seite der Dura dicker und erbabener ist, als auf der Seite des Periosts. Die Zellenthätigkeit ist in derselben sehr lebhaft und geht Haud in Hand mit einem ausserordentlichen Reichthum an nengebildeten Gefässen, so dass in den dem Knochen am nächsten liegenden schichten ihre faserige Structur geradezu verloren gegangen ist und sie wie aus jungen Bindegewebselementen zusammengesetzt erscheint, die sich anf einem ans zahlreichen Gefässschlingen bestehenden Netze angehäuft haben. Was die Dura angeht, so hängt sie völlig an. Ferner ist der Rand des Defects nicht ganz nekrotisch, sondern man sieht dort Inseln und Zonen neugebildeten Knochens, der den Elementen der haversischen Canäle und der Diploë 
seinen Ursprung verdankt. An dem Punkte, wo sich der durch den osteoplastischen Lappen hergestellte Defect befand, sieht man bei einem allgemeinen Ueberblick ein vollständiges faseriges Zellengewebe, reich an Flementen und Gefässen. Dieses verbindet sich einerseits mit der Dura, andererseits mit dem Periost, und Gefässe und Elemente, die aus den haversischen Canälen und den offenen Lücken der Diploë kommen, dringen in dasselbe ein; und mitten in diesem faserigen Cellulargewebe sieht man Lamellen und Knocheninseln.

Bei genauerer und systematischer Prufung erkennt man, dass dieses Cellulargewebe aus Bindegewebsbïndeln besteht, die sich auf allerlei Weise untereinander kreuzen und durch junge Cellularelemente von einander getrennt sind. Diese letzteren stammen grösstentheils aus der Proliferation der Bindegewebszellen, die ursprünglich längs den Bindegewebsbündeln angelagert waren. Die sehr zahlreichen Capillargefässe findet man in diesen $Z$ wischenräumen zwischen den Bundeln von den jungen Bindegewebszellen umgeben, die mehr oder weniger regelmässig in Eiform, mit grossem, abgerundetem Kern angeordnet sind. Solche Bildungen hängen ebensowohl von dem Periost, als von der Dura ab, in welcher letzteren die Zellenthätigkeit wahrhaft erstaunlich ist. Dieses Gewebe drückt und schliesst die oben von uns erwähnten Lamellen zusammen; man sieht, wie sich die Gefässe an diese andrängen, sie streifen, in ihr Inneres eindringen und sie zernagen, während wir sie ringsumher von einer Zone junger, theils eiförmiger, theils polygonaler Elemente mit abgerundetem Kern umgeben sehen. Diese gruppiren sich, wenn auch in etwas unregelmässiger Weise, zu einer ununterbrochenen, aus säulenförmigen Elementen bestehenden Schicht. Wenn einige Lamellen ganz abgestorben und der Corrosion und Absorption unterlegen sind (wie es der Zustand der Knochenelemente, das gesteigerte Lumen der haversischen Canäle und die Gegenwart zahlreicher Gigantenzellen bezengen), findet man in anderen neben abgestorbenen Theilen vollkommen vitale von jungem Knochen, der aus der osteoblastischen Schicht herrührt, von der diese Lamellen, wie gesagt, umgeben sind. Wenn man nun aber diese Erscheinung bei den grossen Lamellen nur an gewissen Punkten bemerkt, so sieht man in den kleinen Lamellen einen nekrotischen centralen, ganz von jungem Knochengewebe umgebenen Theil. Die Scheidelinie dieses nekrotischen Theiles von dem vitalen ist ziemlich scharf. In den verschiedenen Präparaten aus dieser Periode ist diese Erscheinung die vorwiegende. Wenn jedoch in den meisten Fällen die Bildung des neuen Knochens nach dem Modell der fruberen nekrotischen Knochenbruchstucke erfolgt, darf doch nicht tbersehen werden, dass hier und da, wenn auch selten, die Bildung neuen Knochens, unabhängig von den Knochenbruchstücken, anzutreffen ist. So bemerkt man Gefässe im Querschnitt, die alle von jungen Flementen umgeben sind, von denen die am meisten peripherischen mit einem etwas grösseren, uuregelmässigen Kern in wohl abgegrenzten und in einer homogenen Substanz eingeschalteten Räumen gelegen sind. Diese Substanz nimmt ihre Färbung ganz mit den dem Knochen eigenen Tinctionseigenschaften an, während von der Peripherie aus sich andere Osteoblasten anlagern und das Wachsthum des Knochens befördern. 
Kurz zusammengefasst, sowohl Dura als Periost haben an der Wiederherstellung des Defects gleichen Antheil, und die Bildung neuen Knochens durch Uebereinanderlagerung findet vorzugsweise dort statt, wo sich die früheren, vom Operateur zum Zweck des Iappens, wie beschrieben wurde, hervorgebrachten Bruchstücke befinden. Die Xcubildung von Knochen kommt jedoch auch metaplastisch vor, indem sie in gewöhnlicher Weise eine osteoidische Phasis durchmacht. Die Elemente der Diploë und der baversischen Canäle bleiben jedoch nicht ganz unbetheiligt. So sieht man am äussersten Rande der Section von den haversischen Canälen aus in das den Defect ausfüllende Zellengewebe Gefässe eindringen, die ganz von jungen Bindegewebs. elementen umgeben sind, die sich als osteoblastische Elemente an die abgestorbene Knochenoberfläche anlagern und zur Entstehung neuen Knochens Anlass geben. In diesen Präparaten fehlt durchaus der neoplastische Charakter einer Knochenbildung, die eine Knorpelbildungsphase durchläuft, während der ganze Process der Ossification in Wahrheit vermittelst osteoblastischer Elemente zu Stande kommt.

4 Monate. In dieser Periode bemerkt man einen wesentlichen Fortschritt. Fs handelt sich nicht mehr um das Vorwiegen von Zellengewebe, das schon nach 2 Monaten zu bemerken war. Hier wiegen die Knochenbestandtheile vor, die in grossen Schichten angeordnet sind. In einigen derselben tritt der schwammige Cliarakter deutlich hervor, während dieser bei anderen kaum angedeutet ist. Die Structur nähert sich bedeutend der des compacten Knochens an, und die einen wie die andern werden durch das erwähnte, an Elementen und Gefässen reiche faserige Zellengewebe zusammengehalten, das ebensowohl im Periost, als in der Dura seinen Ursprung hat.

Betrachten wir die Lamellen, bei denen der schwammige Charakter weniger ausgesprochen ist, so finden wir die ihnen entsprechenden Theile sowohl des Periosts als der Dura in einem erstaunlich thätigen Zellenbildungsprocess begriflen, und die jungen Elemente von dem gewöhnlichen eiförmigen Typus, mit abgerundetem Kern, sehen wir sich in Balken anordnen, welche, in die Knochenoberfläche implantirt, an ihren entferntesten Theilen der Ossification anheimfallen. Hierauf bilden sie eine durale und eine periostale Schale neugebildeten Knochens, der aus elementenreichen Balken gebildet ist, von denen die am meisten peripherischen die Oberfläche der Balkenhöhlung tberziehen nach Art und Weise des osteoblastischen Häutchens, das wir unter ähnlichen Umständen in den im Wachsthum befindlichen Knochen antreffen. Im centralen Theil der erwähnten Lamellen geht die Knochenbildung in verschiedener Weise vor sich. In der That ist es bei starker Vergrösserung nicht schwer, an dieser Stelle mehr oder weniger weite Strecken zu sehen, in denen der Knochen nicht mehr vital ist, wie sich an den Knochenkörperchen erkennen lässt, die sich erweitert haben und ganz der Elemente entbehren, oder voll von pulverisirtem Detrit sind, oder (in sehr geringer Anzahl) in ihrem Innern ver- 
schrumpfte und entstelite Zellen enthalten. In diesen Zonen erblickt man weite, unregelmässige Canäle (mit grossen parietalen Erweiterungen), in denen sich zahlreiche Capillargefüsschen inmitten eines an Flementen reichen Gewebes befinden. Einige von diesen tragen den Typus von Immigrationselementen an sich, andere den von jungen Bindegewebselementen; die einen wie die anderen uben ihre Thätigkeit in Aushöhlung und Zernagung des nekrotisirten Knochens nach allen Richtungen hin aus, indem sie zur Bildung zahlreicher Gigantenzellen beitragen. Diese Canäle offnen sich an der Peripherie der Lamellen, und das in ihnen enthaltene Gewebe verdankt seinen Ursprung sowohl der Dura als dem Periost, die ihre Ausläufer dahin seuden, so dass die Continuität des Koochens theilweise unterbrochen wird. Der ubrige Theil des Knochens ist vital, wahrer neugebildeter Knochen, der den Typus der haversischen Productionen um ein Gefäss herum trägt; während wir anderswo im Centrum einer concentrischen Knochenbildung einer Höhlung begegnen, in der sich Ueberbleibsel des alten Knochens von nekrotischem bleichen Aussehen, mit vielen Vacuolen befinden, die rings von Cellularelemen-

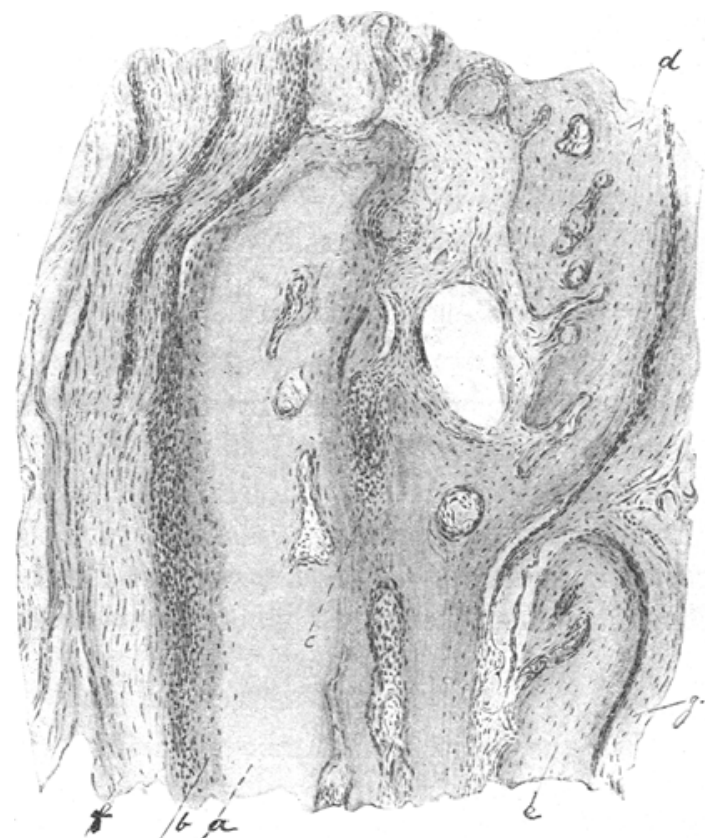

Fig. 4. ten, die sie nach allen Richtungen hin zerstören, umgeben sind, wäbrend utber der Höhle selbst parallele Knochenbildungsprocesse vor sich gehen. Im Grossen und Ganzen wohnen wir hier der lebhaften Zerstörung aller alten nekrotischen Theile bei, die in den ersten Perioden den Mittelpunkt und das Vorbild der Ossification bildeten, während andererseits die Knochenbildung in den oben von uns beschriebenen charakteristischen Zugen vollständig ist. Die einen mehr schwammigen Typus tragenden Theile vertreten fur uns ein vorgeschritteneres Stadium; hier ist die Absorption viel weiter vor sich gegangen und von dem abgestorbenen Knochen sieht man nur noch spuren. Wenn man hier und da noch $A$ bsorptionsprocesse in Thätigkeit findet, wie sich aus der Gegenwart der an einigen Stellen in grosser Anzahl vorhandenen Gigantenzellen entnehmen lässt, so hat man 
doch an den meisten Punkten einen wirklichen Bildungsprocess von Markknochen vor sich, und die osteoblastischen Elemente füllen die Theile der einzelnen IIöhlen aus und tragen zur Bildung neuen Knochens bei, der die Dicke der Balken vermehrt. - Die Dura und das Periost sind bedeutend dicker geworden, und in ihren tieferen Theilen sieht man sie auch hier zu einem ausschliesslich aus Elementen mit einem schönen abgerundeten Kern bestehenden Gewebe zusammengeschmolzen. Dieses Gewebe ist ein gefässreiches Proliferationsproduct der Bindegewebselemente, das in Balken angeordnet ist, die, indem sie sich itber den ursprünglichen Knochen legen, ossificiren und in die Buchten und IIöblen des Knochens eindringen. Dann erblicken wir einen centralen Theil mit den Gefässen und einen aus osteoblastischen Flementen gebildeten peripherischen, die sich an die Wände der genannten Knochenhöhlen anlagern und sie bedecken, indem sie zur Bildung neuer Knochenschichten in der gewöhnlichen Weise beitragen.

An den Knochenrändern des alten Defects ist die Restitutio ad integrum nunmehr vollständig, und, abgesehen von einer grösseren Ausdehnung der haversischen Canäle und einer ziemlich grossen Activität von Seiten der Dura und des Periosts, unterscheiden sie sich in keiner Hinsicht vom normalen Zustand, auch ist ibre Continuität mit den vorher beschriebenen Knochenlamellen vollkommen.

In diesem dritten Experiment ist das makroskopische Aussehen dasselbe, wie im zweiten, d. h. man hat eine völlig ununterbrochene Knocbenoberfläche und von der Oberfläche der Dura sieht man die Furchen und Einsenkungen, die von den Gehirnwindungen hervorgebracht sind, an der Stelle, wo der Defect reparirt ist. Die Dura hängt an. Das Gehirn ist vollkommen frei.

\section{Osteoplastischer Lappen Durante's. - Ohne Dura.}

Für die Experimente habe ich Stücke von 5 Thieren genommen, von denen 4 per priman geheilt waren, während eines an nachfolgender Gehirnentzündung mit Eiterung gestorben war. Von diesem letzteren habe ich ein Stück genommen, weil es Gehirnbruch aufwies, ausserdem, um mich davon zu überzeugen, wie der Lappen sich in diesen Fällen mikroskopisch verhält. Bei den Thieren, die, weil an Shock gestorben oder wegen Eiterung unbrauchbar geworden, bei Seite gelassen wurden, ist zu bemerken, dass von dem Lappen der letzteren nur wenige freie und bewegliche Lamellen iibrig geblieben waren; das Gehirn hing an und ragte über den Defect hinaus. Einen solchen Gehirnbruch habe ich in diesen Fällen immer schnell eintreten sehen.

Die Experimente dauern 25 Tage bis zu 3 Monaten.

25 Tage. Bei der Autopsie bemerkt man an der äusseren Oberfläche des Schädels eine geringe Finsenkung an der Stelle des Defects. Das Gehirn hängt dicht an dem Lappen. Bei einer medianen Section 
sieht man am Defectspunkt ein Gewebe von fibrösem Aussehen, in dessen Innern sich Knochenbruchstïcke befinden. Das Gehirn hängt an; jedoch zeigt es kieine Veränderung, ausgenommen in einer sehr schmalen, oberflächlichen Zone, die sich als erweicht und wie in Brei verwandelt erweist, von kupferrother Farbe.

Mikroskopisch sind die Knocheuränder des Defects, der Defect mit dem osteoplastischen Lappen und das anhangende Gehirn in Betracht zu ziehen.

Die Knochenränder zeigen sich uns in den verschiedenen Sectionen mit einer sehrbeschränkten nekrotischen Zone gerade auf dem Niveau der den Defect ungebenden Oberfäche und in einem intensiven $\mathrm{Ra}$ refactionsprocess und einer activen Absorption in den dem Defect am nächsten liegenden Knochentheilen. Hiermit ist eine lebhafte Reaction der weichen Gewebe der Diploë und der erweiterten haversischen Canäle verbunden, die sich in dem Ueberfluss an Leukocyteninfiltration wie auch in der starken Proliferation der Bindegewebselemente und der ausserordentlichen Menge von neugebildeten Capillaren ausspricht. Die Dura und das Periost, die den Knochen

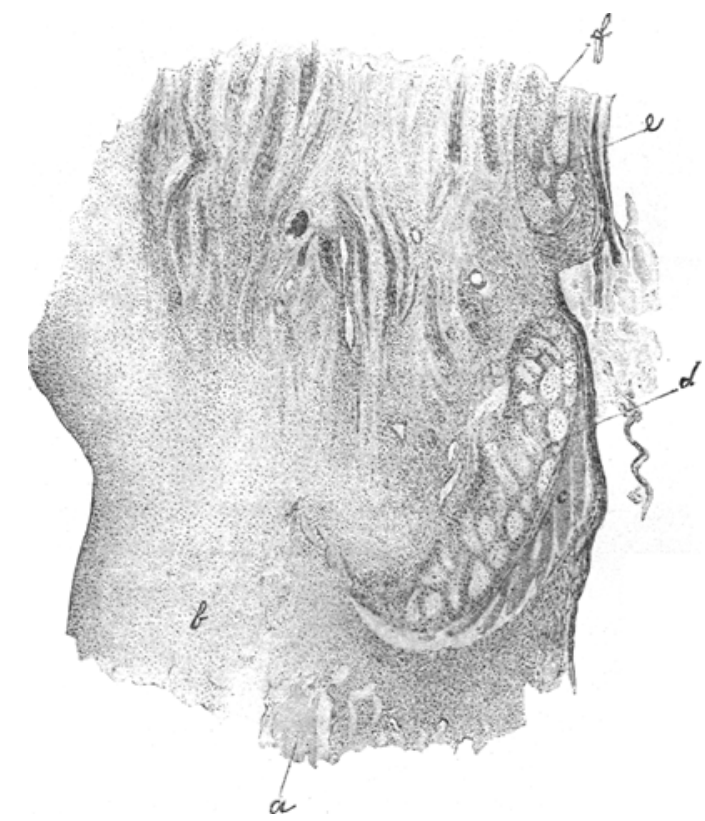

Fig. 5. umbillen, weisen in ihren weiter abliegenden Theilen kaum Spuren von Reaction auf; diese beginnt an der Stelle des Läsionspunktes. Man sieht dann die Elemente, aus denen sie zusammengesetzt sind, in eine lebhafte Wucherung tubergehen, so dass ihr Bau nur noch in den äussersten Theilen sichtbar ist, während sie in den mehr nach innen gelegenen die Structur eines sehr gefässreichen Gewebes annehmen, das fast ausschliesslich aus grossen Elementen mit abgerundetem Kern besteht, die jungen Bindegewebselementen entsprechen. Die Dura bricht auf dem Niveau des Läsionspunktes $a b$, um sich in Form eines fibrösen Zellengewebes oben umzubiegen, oberhalb des Knochenrandes, und um sich mit entsprechenden, vom Periost herruhtrenden Neubildungen zu verschmeizen. Das Periost biegt sich nach unten um, in der Richtung nach den weichen Meningen, die verdickt sind und sich an das faserige Zellen- 
gewebe schliessen, sich eng mit demselben verbindend. Dies letztere hat die gewöhnlichen Charakterzlige eines aus Bindegewebsbtundeln gebildeten faserigen Gewebes, an welches junge Bindegewebselemente angelagert sind, die in mehr oder weniger grosser Frequenz und mehr oder weniger regelmässig die Gefässschlingen nmgeben, die vom Periost aus in den Defect eindringen, indem sie die cellulären Neubildungen begleiten. Gerade in der Nachbarschaft des den Rand des Defects bildenden Knochens sehen wir diese Elemente von vorzugsweise periostalem Ursprung sich in feinen Bälkchen anordnen, die, indem sie in Knochen thergehen, sich mit den Enden der nekrotischen Binde verknüpfen. Sie unterscheiden sich jedoch völlig von dieser, da sie in den Sectionen das Aussehen eines feinen und eleganten netzförmigen Gebälkes annehmen, das bis in die von der Operation her offen gebliebenen Litcken der Diploë eindringt. Vielleicht tragen zur Bildung desselben auch die Elemente der Diploë selbst bei. - Im ubrigen Theil des Defects sehen wir uberall das gewöhnliche, an cellulären Elementen reiche Gewebe, das dem vorher beschriebenen entspricht, und unzweifelhaft periostalen Ursprungs ist. Dieses umhallt rings die nekrotischen Knochenbruchstucke des Lappens, bei denen der Rarefactions- und Absorptionsprocess nur durch die grössere Anhäufung von Elementen an ihrer Peripherie ange. deutet wird, während sie selbst wie träge und fremđartige Körper dastehen. Anders verhält es sich mit einigen feinen Brnchstiłcken, die sich der periostalen, osteogenetischen Schicht näher befinden. Von der Seite des Periosts sieht man dann ein vollkommenes System von neugebildeten Knochenbalken, die sich an die Bruchstïcke des alten Knochens anlegen. Diese lediglich periostale Neubildung von Knochen ist ziemlich lebbaft, obwohl sie viel weniger ausgesprochen ist, als in kürzeren Perioden, wo die Dura unverletzt ist.

Beim Gehirn haben wir keine anderen Verletzungen, als in den oberflächlichsten Schichten der moleculären Zone, in denen die Structur des Mantels nicht mebr erkennbar ist. Es fehlen die Nervenelemente, vielmehr wiegen die leukocytären Infiltrationselemente vor. Zablreiche Gefässschlingen geben von den weichen Meningen in's Innere des Gehirns und bringen so schwache Adhäsionen zwischen diesem und den weichen Meningen hervor, die sehr verdickt, reich an Elementen und uberaus gefässreich sind.

30 Tage. Der makroskopische Befund ist in den Hauptpunkten mit dem vorhergehenden identisch.

Die mikroskopischen Thatsachen sind ebenfalls von den in der vorhergehenden Periode beschriebenen wenig verschieden.

Auch hier findet die, sei es periostale oder durale Reaction längs der die Verletzung umgebenden Knochen nur gerade auf dem Niveau der Verletzung selbst mit denselben oben beschriebenen charakteristischen Eigenthümlichkeiten statt. Sehr klar ist hingegen das Verhalten der Dura, die, an der Continuitätsunterbrechung des Knochens angelangt, dort, wo sie abgeschnitten ist, sich um die Ränder schlägt und mit den Neubildungen des Periosts zusammentrifft. Man sieht, wie diese letzteren sich von dem oberen Rande des Defects aus uber die Neubildungen der Dura ziehen, 
sich erweitern und über den ganzen Defect verbreiten; auch hier begegnen wir den gewöhnlicben charakteristischen Zügen eines an Elementen und Gefüssen sebr reichen Bindegewebes. Die osteogenetische Zone sticht durch ihren ausserordentlichen Reichtluum an Zellen mit grossem, rundem Kern hier ebenso hervor. Diese bilden eine ziemlich dicke Schicht, aus der sie sich hier und da ablösen, um die Gefässe zu begleiten, indem sie sich in Balken gruppiren. Diese stützen sich, während sie sich nach und nach in Knochen verwandeln, mit ibren äussersten Enden auf die vom Lappen ubrig gebliebenen völlig nekrotischen Knochenlamellen. Hier wohnt man, ausser dieser durch eine osteoidische Phase hindurchgehenden balkenförmigen Knochenbildung, einer Neubildung von Knochen durch Uebereinanderlegung bei, die sich in diesem Moment, kann man sagen, rings um die der osteogenetischen Schicht am nächsten liegenden nekrotischen Lamellen vollziebt. Diese werden in diese Neubildung mit hineingezogen, zu der auch die osteoblastischen Elemente beitragen, welche sich, vom Periost aus die Gefässe begleitend, zu einer mehr oder weniger dicken Schicht aneinanderlegen, und zwar rings um die nekrotische Lamelle, in der, was hervorgehoben zu werden verdient, der Rarefactions- und Absorptionsprocess ziemlich weit vorgeschritten ist. Dieser Erscheinung begegnen wir auch auf eiuer kurzen Strecke an den Knochenrändern, jedoch mit einer an vielen Punkten bemerkbaren Andeutung von Wiederherstellung vermittelst der diploischen Elemente.

30 Tage. Bei diesem Thier fand, wie oben gesagt, Eiterung und Tod in Folge von Meningoencephalitis statt. Von dem Lappen ist nur eine Spur in kleinen, beweglichen oder völlig freien, angefressenen Lamellen übrig; so ist der Defect offen und durch ihn bricht das von einer dicken, fibrinösen und eiternden Schicht bedeckte Gehirn hindurch.

Die Ränder der Wunde sind einer in hohem Grade activen Rarefaction ausgesetzt, weshalb die Diploëräume und die baversischen Canäle sich sehr erweitert finden. Die Knochensubstanz ist an einigen Punkten nekrotisch, an andern dagegen vollkommen erhalten, jedoch bemerkt man keine Andeutung von Neubildung, weder von Seiten des Gewebes, das die diploischen Räume ausfüllt, noch von Seiten des Periosts, die beide infiltrirt sind. Die Dura ist, soweit sie existirt, sehr verdickt. Von dem osteoplastischen Lappen sind nur wenige Knochenbruchstiicke übrig, die in ein phlogistisches Gewebe verwickelt sind. Ein grosser Theil des Defects ist von den weichen Meningen besetzt, die infiltrirt und verdickt sind und eiternde Stellen aufweisen, sowie vom Gehirn, das sich gleichfalls als infiltrirt und hyperämisch erweist und an den oberflächlichsten Schichten der Degeneration verfallen ist. In Folge davon ist der Nervonbau kaum erkennbar. In der That, während dort die Infiltrations- und Eiterungselemente sehr zahlreich sind, fehlen diejenigen der Nerven ganz, oder sind einer bedeutenden nuclearen Alteration ausgesetzt. In den unteren Schichten 
ist in Beziehung auf den cellulären Theil nichts hervorzuheben, abgesehen von einer mässigen Infiltration, einer ziemlich starken Hyperämie und einer Vermehrung der perivasalen und pericellulären Lymphräume.

40 Tage. Makroskopisch ist keine Verschiedenheit vom ersten und zweiten Experiment dieser Serie zu verzeichnen.

Die Thatsachen, denen wir hier begegnen, bezeugen eine beträchtliche Verzögerung in der Entwicklung des Reparationsprocesses, in Vergleich mit dem, was wir am 25. und 30. Tage zu beobachten, Gelegenheit fanden. Die Knochenränder zeigen nur eine sehr schmale, unmittelbar auf dem Niveau der Sectionsoberfläche liegende, nekrotische Binde. Auch die Rarefactionsprocesse, die wir in den andern Präparaten an dieser Stelle immer sehr ausgesprochen fanden, sind hier kaum angedeutet. Die Activität der Dura beginnt in der Nähe der Sectionslinie und macht sich durch eine beträchtliche Verdickung und eine Vermehrung der Gefässe und dẹ Zellenelemente bemerkbar. Von einem solchen Gewebe, das alle Eigenthümlichkeiten eines jungen Bindegewebes an sich trägt, findet allmählich, gerade auf dem Niveau des Defeets, der Uebergang zu einem Gewebe vom Typus eines ausgewachsenen Bindegewebes statt. Dieses verbindet sich mit der periostalen Neubildung, die an den meisten Stellen gleichfalls den Typus von ausgewachsenem Bindegewebe annimmt, welches reich an Gefässen und spindelförmigen Elementen ist. Dieses Bindegewebe umhüllt die nekrotisirten Knochenbruckstücke des Lappens rings umher und zehrt dieselben nach allen Richtungen auf, indem es die Bildung zahlreicher Gigantenzellen hervorruft. Bestimmte und ausgesprochene Ossificationserscheinungen sind an keiner Stelle vorhanden; man sieht nur hier und da die periostalen Proliferationselemente, balkenförmig angeordnet, in Richtung auf die näher gelegenen Knochenbruchstücke vorschreiten; jedoch, obwobl ich verschiedene Colorationen angewandt habe, ist es mir nicht gelungen, an irgend einem l'unkt auch nur einen Anfang von Knochenbildung zu entdecken.

3 Monate. Die Continuität des Knochens ist an der Aussenseite vollkommen; das Gehirn hängt an; die Dura bildet auf dem Niveau des Defects eine Art von Wulst. Was die Ränder der Wunde anbetrifft, so erscheinen sie in verschiedenen Präparaten durchaus in normalem Zustand. Hervorzuheben ist nur ein ziemlich vorgeschrittener Grad von Sklerosis, in Folge deren die Diploühöblen bedeutend zusammengeschmolzen oder geradezu verschwunden sind. Vorwiegend ist der lamellenartige, haversische Typus, als ob die Sklerosis von Seiten der der IDiploë eigenen, osteoblastischen Elemente auf concentri- 
sche Weise stattgefunden hätte. Wenn wir nach und nach von den entfernteren Theilen aus nach dem Defect vorschreiten, fangen wir an, nekrotische Knocheninseln zu bemerken, die ringsumher von der Knochenneubildung ergriffen und zusammengeschnürt sind. Diese Neubildung unterscheidet sich von dem früheren Knochen durch den weniger gleichförmigen und intensiveren Farbenton, den sie angenommen hat; durch den Typus der wohl hervortretenden und färbbaren Knochenelemente und dadurch, dass sie mit dem periostalen osteogenetischen Gewebe direct in Zusammenbang steht, wie sie sich auch vom abgestorbenen Knochen unterscheidet, weil dieser klarer vacuolisirt und körnig ist und weniger die Farben annimmt. Diese Erklärung wird durch die Betrachtung anderer kleiner nekrotischer Bruchstücke bestätigt, die, von der Continuität des Knochens getrennt, von einer in Ossification befindlichen osteoblastischen Zone umgeben sind, während andere, schon von einem Streifen jungen Knochens umgeben, sich mit dem früheren Knochen verbinden. Auf diese Zone folgt eine andere, aus feinen und eleganten Knochenbalken gebildete, die den früheren alten Knochen mit dem neugebildeten Theil, der den Defect ausfüllt, wie eine Brücke verbinden. Bei einem allgemeinen Ceberblick stellt sich diese Zone als aus mehreren Stücken von verschiedener Grösse bestehend dar, die untereinander, sei es durch ein faseriges Zellengewebe, das vom Periost herkommt und die untere Oberfläche nach dem Gehirn hin bedeckt, oder durch feine Knochenbalken verbunden sind. Oben haben wir das Periost, dessen osteogenetische Schicht in lebhafter Proliferation begriffen ist, indem sie zu der Bildung von Balken beiträgt, die sich, indem sie sich ossificiren, an die früheren anlegen, so dass jede einzelne Lamelle von der periostalen Seite aus im Wachsthum begriffen ist. Auf der Seite des Gehirns befindet sich eine feine, faserige Schicht Bindegewebe, die reich an Gefässen und schönen, eiförmigen Elementen mit rundem Kern ist, welche letzteren, an die Lamellen ringsumher in ununterbrochener Schicht angelagert, zu deren Wachsthum beitragen, oder Bruchstücke umhüllen, die sie zusammendrücken und zu Centren neuer Knochenbildung machen.

Unter diesem Bindegewebe befinden sich die weichen Meningen; sie sind ein wenig verdickt und hängen eng an der Gehirnmasse an. Ueberall begegnen wir mannigfaltigen Ossificationssystemen, und während auf der Seite des Periosts der balkenförmige Typus (mit eng aneinander liegenden Balken) überwiegt, bemerkt man an anderen Stellen concentrische Anlagen rings um ein Gefäss oder ein Knochenbruchstïck. Iiese Knochensplitter zeichnen sich durch ihre scharfe 
und präcise Demarcationslinie aus und sind grösstentheils der Zernagung ausgesetzt. Der allgemeine Typus dieser, den Defect ausfüllenden Neubildung von Knochen ist der des compacten Knochens.

Wir haben erwähnt, dass dic Gehirnmasse eng an den weichen Meningen anhängt; diese senden zahIreiche Gefässschlingen in's Innere des ( Kehirns aus. Das letztere ist jedoch vollkommen unversehrt, sowohl seinem allgemeinen Ausseben nach, als in seinen Einzelheiten, abgesehen von einer sehr schmalen pheripherischen Zone, wo die Structur der Nerven nicht mehr erkennbar ist und die Elemente fehlen, während einige Leukocyten in ibr Inneres eingewandert sind.

Die Ossification greht also lebhaft vor sich, sowohl im Fall, wo die Dura unversehrt bleibt, als in demjenigen, wo sie fortgenommen ist, und schreitet schnell zur völligen Restitutio ad integrum vorwärts. Es ergiebt sich jedoch aus unseren Versuchen eine Thatsache, nämlich dass in den ersten Perioden, bei fehlender Dura, die Knochenbildung weniger schnell stattfindet, im Vergleich zu dem, was wir bei unversehrter Dura, selbst in kürzeren Perioden, bemerken. Da sich diese Erscheinung in den drei ersten Experimenten der zweiten Serie immer unverändert wiederholt, muss ich wirklich annehmen, dass hier ein wichtigeres und bestimmteres Moment in Frage kommt, als es die individuellen Umstände der Versuchsthiere sind, auf die man sich etwa berufen könnte. In dieser Meinung bin ich noch dadurch bestärkt worden, dass sich die ersten Anfänge der Ossification in unmittelbarer Nähe des Periosts vollzogen. Die Dura spielt also eine Rolle bei der Restitutio ad integrum der Schädelknochen, und zwar als ein wesentlicher Factor. Sicherlich ist sie aber nicht unumgänglich nothwendig und die Experimente liefern den Beweis, dass, wenn auch in den ersten Perioden eine kleine Verzögerung stattfindet, der Process nachher schnell bis zur völligen Restitutio ad integrum verläuft. - Was die Knochenbruchstücke betrifft, die wir am Periost haben anhängen lassen, so hat sich ergeben, dass diese absterben und keinem anderen Zweck dienen als dem, die Knochenbildung zu dirigiren und eine Function als Modell auszuüben, um die Ossification anzustacheln. Auf Grund unserer histologischen Befunde können wir dies mit vollkommener Gewissheit behaupten, da wir Gelegenheit gehabt haben, festzustellen, dass die Knochenbildung ohne diese nekrotischen Knochenstückchen bei dem gewöhnlichen Process nur selten und nur in fast ausserordentlicher Weise statthat. Diese Bruchstücke bilden Ossificationscentren, die in der Folge der völligen Absorption verfallen und verschwinden, um Gefässen und Elementen periostalen oder duralen Ursprungs Platz zu machen, die dann ihrerseits jene Phasis centraler 
Knochenbildung einleiten, durch welche der neugebildete Knochen aus einem schwammigen in einen compacten übergeht, in Erwartung der letzten Involutionsphasis, der wir bei dem von uns studirien Lappen des Menschen beiwohnen und die zur Gewichtsverringerung des normalen Knochens und zur Wiederherstellung des physiologischen Typus führt. So wiederholt sich mit mathematischer Genauigkeit, was sich bei den gewöhnlichen Knochennarben constatiren lässt.

In Bezug auf die Gefahr eines Gehirnbruches muss ich erklären, dass dieselbe bei fehlender Dura durchaus nicht existirt. In dieser Ilinsicht stimmt die Erfahrung des Laboratoriums mit den klinischen Resultaten überein. Denn es ist ein klinisch unbestreitbarer Grundsatz, dass Gehirnbruch nur bei phlogistischen und neoplastischen Erscheinungen im Gehirn vorkommt, es sei denn, dass man jenes unbedeutende Hineinragen des Gehirns in den Defect, das sich sogleich in den ersten Perioden bei Unterbrechung der Dura beobachten lässt, als Gehirnbruch betrachten will. Diese letztere Erscheinung führt mich zur Annahme, dass hierin vielleicht einer der Coëfficienten jener Verzögerung zu suchen ist, die wir in den Anfangsperioden der zweiten Serie der Experimente beobachtet haben.

Temporäre Resection des Knochens. Reimplantationdes resecirten Knochens.

Die von mir bei den Experimenten befolgte Methode war die folgende: zuerst ein Einschnitt in krummer Linie durch die ganze Dicke bis auf den Knochen, wie gewöhnlich in der temporoparietalen Gegend, dann Befreiung des Periosts von dem Knochen. Bei einigen Thieren habe ich eine Trepankrone von $3 \mathrm{~cm}$ Durchmesser angewandt, indem ich eine Scheibe, die ich sogleich wieder reimplantirte, ausschnitt. Auf diese Weise habe ich 6 Thiere operirt, von denen nur ein einziges per primam geheilt ist, während, trotz aller meiner Vorsichtsmaassregeln, alle anderen der Eiterung verfallen sind. Ich dachte daran, dass dies vielleicht der Misshandlung des Knochens durch den Trepan zuzusehreiben sei, daher entschloss ich mich, meine Technik zu verändern und operirte andere 5 Thiere mit einem schneidenden Instrument. - Mit einem sehr feinen Hohlmeissel habe ich ein rechtwinkliges Knochenstück mit einer! Basis von $31 / 2 \mathrm{~cm}$ auf $2-21 / 2 \mathrm{~cm}$ Höhe abgeschnitten und die Continuität auf 3 Seiten unterbrochen, indem ich dem Schnitt mit dem Meissel eine möglichst schräge Richtung gab, damit er als Verbindungsglied dienen könnte; dann brachte ich mit einem Elevatorium oder einem starken Meissel auf der nicht unterbrochenen, aber sehr verdünnten Seite den Bruch 
hervor, der vollkommen und geradlinig ausfiel. Auf diese Weise vermied ich, dass das Stück limuntersinken konnte und erreichte, dass der Defect so weit als möglich bedeckt blieb; darauf nähte ich die ganze Masse der weichen Theile zu einer Lage zusammen. Von diesen so operirten 5 Thieren heilten nur 2 per primam; die andern 3 heilten anfangs per primam, aber bald darauf (zwischen dem 7 . und 15. Tage) stellte sich ein Abscess ein, in Folge dessen der Versuch negativ ausficl. Zwei von diesen der Eiterung ausgesetzten Thieren conservirte ich, das eine 30 Tage, das andere 3 Monate lang, indem ich sie zu heilen suchte, so dass bei dem von 3 Monaten nur ein ganz kleiner Fistelgang, der sich bis auf den unbedeckten Knochen erstreckte, übrig geblieben war. Bei der Autopsie fand ich in beiden Fällen den Discus von den weichen Theilen entblösst, frei beweglich und nekrotisch (in dem Fall von 3 Monaten war er zernagt und verkleinert). Der Discus war kreisförmig von Granulationen umgeben, die von dem Defect ausgingen, welcher gleichfalls mit identischen Granulationen bedeckt war. Ausser an den Hunden wiederholte ich das Experiment mit derselben Technik an den Kaninchen. Von diesen operirte ich drei, bediente mich jedoch zur Unterbrechung der Continuität des Knochens einer ${ }_{\mathrm{b}}^{q}$ Scheere mit kurzen und feinen Schneiden. Auf diese Weise erhielt ich eine sehr kleine Sectionslinie und konnte den Defect nach Gefallen ausdehnen, indem ich immer dafür Sorge trug, dass derselbe in keinem Fall weniger als 2-21/2 cm weit war.

Erstes Experiment. Neunter Tag. Junges Kanincben. Craniektomie links nach der oben beschriebenen Methode. Makroskopisch zeigt der Schädeldiscus keine charakteristischen Merkmale. Aber bei der histologischen Untersuchung sieht man, dass er völlig nekrotisch ist. In der That, was uns auf den ersten Blick auffällt, ist der fast absolute Mangel an Zellenelementen und die Knochenkörperchen stellen sich uns als grossentheils leer dar, oder voll von einem pulverigen Detrit. An einigen Stellen sieht man hier und da noch Spuren von Knochenzellen; bei starker Vergrösserung jedoch bemerkt man an denselben tiefgehende Alterationen. Einige sind verblichen, mit homogenem Protoplasma, ohne noch eine Spur vom Kern zu zeigen, gleich Schatten, andere dagegen entstellt, verschrumpft und mit grobkörnigem Protoplasma, in Cariolysis. Von den haversischen Canälen sind einige leer, andere, ebenso wie die Diploë. böhlen, mit zackigen, unregelmässigen Umrissen und erweiterter, als im normalen Zustande. Sie zeigen eine reiche Infiltration von Leukocyten und Gigantenzellen. An audern Stellen sieht man dagegen inmitten von Infiltrationselementen andere Elemente mit grossem Kern, die spindelförmig oder polygonal sind. Diese berlecken die Wände der Canäle entweder in vielfacheu Schichten oder in einer einzigen Schicht, und immer in einer gewissen Anordnung. - Die Dura und das Periost sind verdickt und reich an Zellenelementen, unter denen man einfache Infiltrations- 
elemente und andere mit grossem Kern und von Bindegewebstypus unterscheidet. - Wenn wir unsere Aufmerksamkeit auf den Punkt richten, wo die Knochencontinuität unterbrochen wurde, finden wir ein an Gefässen und spindelförmigen Zellen reiches Gewebe, das vom Periost und von der Dura herrührt. In diesem Gewebe ist eine balkenförmige Anordnung angedeutet und es bedeckt die Oberfächen des Bruches, indem es sich mit den analogen Producten der haversischen Canäle und den von der Operation her oflen gebliebenen $\mathrm{Di}$ ploëhöhlen verbindet.

In dieser Periode überwiegen, wie man sieht, die Corrosionserscheinungen iber die Substitutionserscheinungen, die jedoch, was nicht zu übersehen ist, schon jetzt klar ausgesprochen sind.

19 Tage. Grosses Kaninchen. Kraniektomie links Defect von $3 \mathrm{~cm}: 2 \mathrm{~cm}$. Makroskopisch ist kein Unterschied $\mathrm{zwischen}$ dem reimplantirten und dem ursprunglichen Knochen zu bemerken; die Continuität ist völlig hargestellt.

Bei der histologischen Untersucbung ergeben sich interessante Eigenthumlichkeiten. In

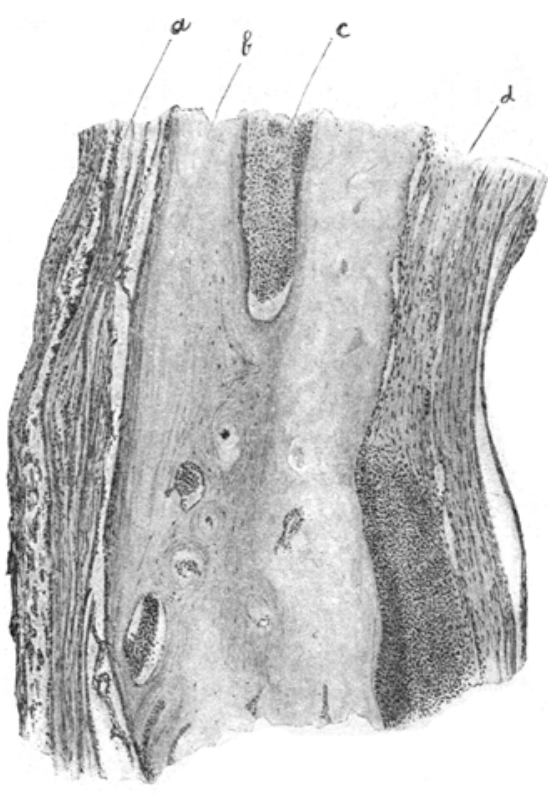

Fig. 6. der That, während der reimplantirte Discus vorwiegend nekrotisch erscheint, zeigen sich an einigen Stellen vitale Zonen. Jedoch bei einer auch nur oberflächlichen Beobachtung erkennt man sogleich, dass keine scharfe Scheidelinie zwischen diesen und dem nekrotischen Theil vorhanden ist, sowie dass dieselben mit der Gegenwart von Osteoblasten zusammenhängen, die palissadenförmig die alten erweiterten baversischen Canäle und die Diploëhöhlen, in denen die Knochenmetamorphose evident ist, umgeben. Auch bier wiegen die Corrosionserscheinungen vor, wie es die Unregelmässigkeit und Erweiterung der in geschlängelter Form auftretenden haversischen Canäle, die reichliche Infiltration und die Gegenwart von Osteoklasten bezeugen. Auf der Seite des Periosts und der Dura, die am nekrotischen Discus anliegen, zeigt sich tberall eine Binde, die aus jungem Knochen, der von dem letzteren scharf unterschieden ist, und aus osteoidischen, in Ossification begriffenen Balken besteht.

20 Tage. Kleiner junger Hund. Der makroskopische Befund ist mit dem vorbergebenden identisch, Die Resection der Knochenscheibe wird mit dem Hohlmeissel ausgefuhrt.

Die Erscheinungen sind beinahe unverändert. Es ist jedoch za bemerken, dass das reimplantirte nekrotische Stilck einer höchst lebhaften 
Absorption unterworfen ist, in solchem Grade, dass von demselben eigentlich nur feine Streifen übrig geblieben sind, die grosse Höhlen um. geben. Diese letzteren sind mit Elementen, vorzugsweise Immigrationselementen und zablreichen Gigantenzellen angefullt. Von den beiden Ossificationen ist die periostale bei Weitem mehr entwickelt, als die durale, trotzdem, dass die Reaction auch auf der Seite der IDra selır lebhaft ist.

1 Monat. Kleiner Hund. Die Knochencontinuität ist vollkommen, sowobl bei der Inspection als bei der Palpation, an der Oberfläche der Dura, wie auf Seiten des Periosts, das völlig anbängt. Der Discus warde

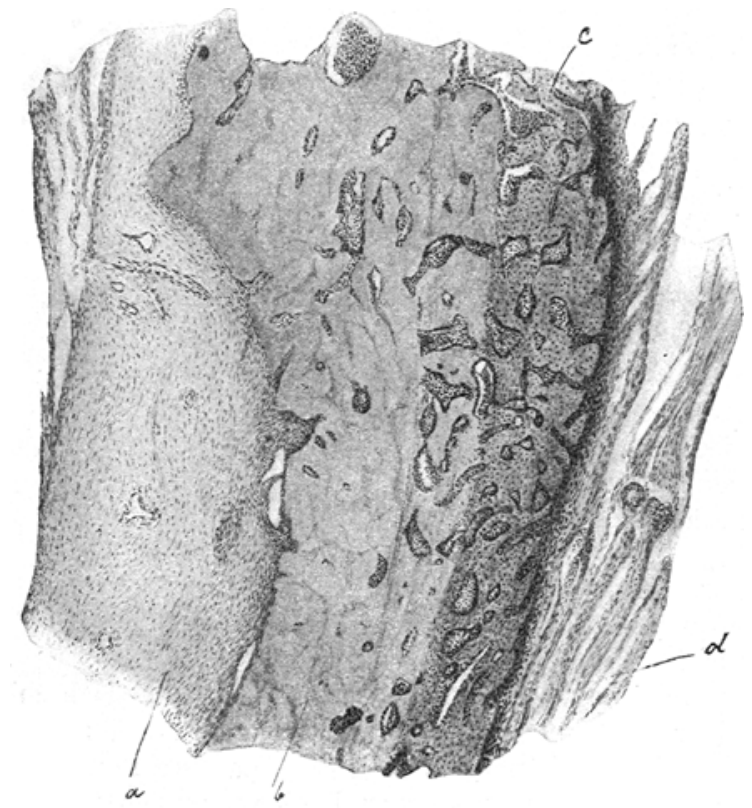

Fig. 7 . mit dem Trepan gemacht. - Géht man von den die Continuitätsunterbrechung begrenzenden Rän dern aus, so findet man an dieser Stelle den Knochen grösstentheils normal. In seinem Innern bemerkt man weiche, von Osteoblasten erfullte Lucken; nur an den der Continuitätsunterbrechung zugewendetenPunkten befindet sich eine dinne nekrotische Binde. Rings um diese umher, wie auch um die durale und periostale Oberfläche des Randes, sieht man an dem ursprünglichen Knochen eine vollstän-

dige, neugebildete Balkenformation, die sich durch bleichere und weniger gleichmässige Färbung, sowie durch grosse und klar hervortretende Elemente auszeichnet, und sich eng an den ursprünglichen Knochen anschliesst, so dass dieser unregelmässige, geschlängelte Umrisse zeigt, mit Einbuchtungen, in die sich die Gefässschlingen legen, welche von der Dura und dem Periost ansgehen, und von Immigrations- und eiförmigen Elementen mit grossem, rundem Kern (wahren jungen Bindegewebselementen) umgeben sind. Diese gruppiren sich an der Peripherie in eine Schicht, die die Oberfliche des Balkens bedeckt, und nehmen eine cubische und polygonale Form an. Hier und da hat man wirklich die Histogenesis des Knochens vermittelst dieser Elemente vor Augen, während in der That die Knochenfarbe sich über das benachbarte Zellengewebe verbreitet. Fast als ob eine verschiedeue, von dem intercellulären Theil angenommene 
Natur angedeutet werden sollte, sieht man die Ostcoblasten allmählich in diese neue Grundsubstanz verwickelt.

Identische Erscheinungen bieten sich rings um das reimplantirte Knochenstuck dar, an welchem sich, sowohl auf der Seite der Dura, als auf der des Periosts, uber die ganze Ausdehnung hin die gewöhnliche in Sklerosis und Wachsthum begriffene Balkenbildung neuen Knochens befindet, wie es die sie bedeckenden Osteoblasten und die beginnende Ossification bezeugen, die auf anderen Pankten im Schooss der cellulären Neubildung stattfindet, welche von der Dura und dem Periost, in osteoidischen Balken angeordnet, ausgeht. Diese auf Seiten der Dura und des Periosts vorgehende Thätigkeit ist wahrbaft erstaunlich und bildet einen Contrast zu dem reimplantirten Knochen, der ganz abgestorben und lacnnärer Corrosion anbeimgefallen ist. So haben wir die Erscheinung, dass das wieder eingesetzte Knochenstuck abstirbt und der Absorption entgegengeht, während sich an seiner Peripherie ein ganz neues Knochengewebe bildet, das sich mit der Tendenz zur Sklerosis entwickelt und dazu bestimmt ist, das erstere in Zukunft zu ersetzen. - Dieser Theil ist mit den Rändern des Defects durch das Zwischenglied eines an Elementen und Gefässen reichen faserigen Zellengewebes verbunden, welches sich vom Periost nach der Dura hinzieht.

30 Tage. Die makroskopische Beschreibung des Stilckes ist früher gegeben worden. Ich bemerke nur, dass es mit dem Meissel hergestellt wurde und dass am 8 . Tage Fiternng eintrat.

Mikroskopiscb bemerken wir keine histologische Eigenthümlichkeit, die für unsere Thesis von besonderem Interesse wäre; denn da das reimplantirte Knochenstück abgestorben ist, so befindet es sich geradezu versenkt in eine Zone phlogistischer Neubildung, die es umgiebt und sich einerseits uber die parostalen Gewebe verbreitet, während sie andererseits auf dem Niveau der Dura abbricht. Diese Neubildungszone ist ausserdem sehr verdickt und hat ein fibröses und umsomehr sklerotisches Aussehen, je weiter sie nach nnd nach bis zum Gehirn in lamellenartiger Structur vorrückt. Das Gehirn weist nur einen beträchtlichen Grad von Infiltration mit einer ziemlich starken Hyperämic verbunden auf. Von Knochenreparation findet sich keine Spur, weder auf dem Niveau des Defects, noch an den Knochenrändern. -

$3 \mathrm{M}$ on at e. Fur die makroskopische Beschreibung gilt dasselbe, wie für die vorhergehende. - Eiterung am 15. Tage. - Mit dem Meissel gemacht.

Die hier vorgehenden Erscheinungen sind im Wesentlichen dieselben. Der reimplantirte Knochen ist vollständig sequestrirt und der Defect ist ganz von einem an Elementen und Gefässen reichen, faserigen Bindegewebe ausgefullt. Hier sind jedoch zwei Thatsachen zu bemerken, und zwar einerseits die völlige Unversehrtheit der Ränder, die aus durchaus normalem Knochen gebildet sind, mit weiten, von Osteoblasten besetzten Höblen; andererseits die Neubildung von Knochen, die längs den unteren Theilen des Randes selbst von Seiten der Dura erfolgt. - Ausserdem ist noch eine besonders interessante Einzelheit zu erwähnen. Betrachten wir nämlich die durale Neubildung, so sehen wir in ihrer Mitte, ganz im Inneren, eine nekrotische Knochenschicht, um welche sich rings eine von 
den Osteoblasten hervorgebrachte Binde jungen Knochens absetzt, deren Ränder sich mit denen des nekrotischen Knochens allmählich verschmelzen.

2 Monate. Vollkommene Continuität, sowohl auf der Seite der Dura, als auf der des Periosts. Die Dura hängt an. Mit dem Meissel gemacht.

In den verscbiedenen Sectionen sieht man den ursprunglichen Koochen an den Rändern durchaus normal; es ist nur eine dünue, nekrotische Binde, die in vielen Präparaten fehlt, ubrig geblieben, gerade auf dem Niveau der Sectionsoberfläche. - Periost und Dura befinden sich in lebhafter Reaction und sind verdickt; auf dem Niveau des Defects angelangt, dehnen sie sich aus und verbinden sich zu einem einzigen, faserigen

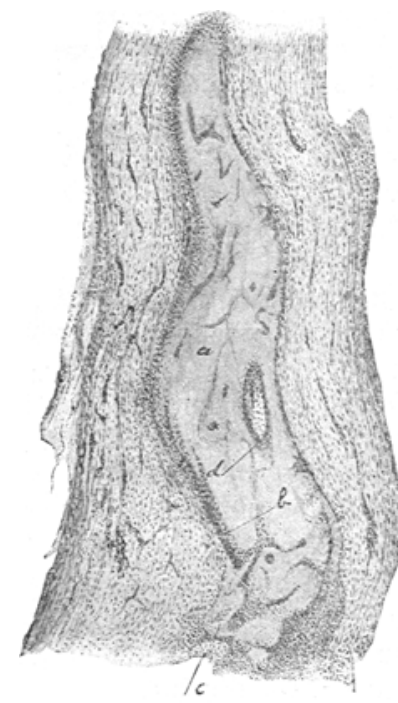

Fig. 8 . Bindegewebe, das den Defect ausfüllt und die Dicke des Knochens genau wiederbolt. Dieses Bindegewebe ist reich an Elementen mit eiförmigem, spindelförmigem Kern und an Gefässen. In einigen Präparaten finden wir gerade in der Mitte desselben eine kleine dünne nekrotische Knochenschicht, die nach allen Richtungen hin von einer Zone lenkocytischer Elemente zernagt wird, die sie umgeben und von allen Seiten aufzehren. Diese Zone stellt das Teberbleibsel des reimplantirten Knochenstuckes dar. - In anderen Präparaten ist dieses Knochenstück mit einer ganzen Neuproduction von Knochen verbunden, die sich am Rande des nekrotischen Theils aufschichtet und nach dem Innern des Defects hin vorschreitet, bis sie das nekrotische Fragment erreicht und dasselbe theilweise umschliesst. In diesem Experiment ist das osteogenetische Vermögen des Periosts und der Dura ohne $Z$ weifel höchst gering und auf die Ränder beschränkt. Die zerstörende Thätigkeit uberwiegt die neubildende.

$21 / 2$ Monate. Weisses Kaninchen. Kraniektomie wie in den vorhergehenden Fällen ausgeftuht. -

Makroskopisch lässt sich nichts beobachten, was die Unterscheidung des reimplantirten Theiles von den Rändern der Section erlaubte. Die Continuität ist vollkommen; man sieht nur einen fast unmerklichen Streifen feiner Gewebe, der sich von der Dura nach dem Periost hinzieht; er scheint dazustehen, als ob er an den Punkt, wo die Continuität des Knochens unterbrochen wurde, erinnern sollte.

In den Präparaten dieser Periode siebt man den Verlauf des ganzen. Reparationsprocesses klar vor sich.

Man bemerkt in der That, dass die Diploëhöhlen und die haversisehen Canäle des ehemaligen Krochens alle von schönen grossen Zonen jungen Knochens umgeben sind, die sich vom alten nekrotischen Knochen durch eine sehr scharfe Demarcationslinie unterscheiden. Auf diese Weise verbleiben von dem reimplantirten Discus nur nekrotische Streifen, die 
nach allen Richtungen hin von den haversischen Canälen durchlaufen werden. Diese sind erweitert und varicös; einige sind schon ganz durch Knochen ersetzt, bei anderen dagegen ist die Ossification noch im Werden begriffen. Man sieht sie von dünnen Zonen jungen Knochens umgeben, während sich in ihrem weit aber das Normale hinaus erweiterten Lumen das ganz von einer dichten Zone von Osteoblasten umringte Gefäss befindet. Diese Osteoblasten gruppiren sich an der Peripherie in concentrische Zonen. Charakteristisch ist die Anordnung, in der die haversischen Neubildungen je nach der Art des Schnittes auftreten. In der That selen wir gerade in der

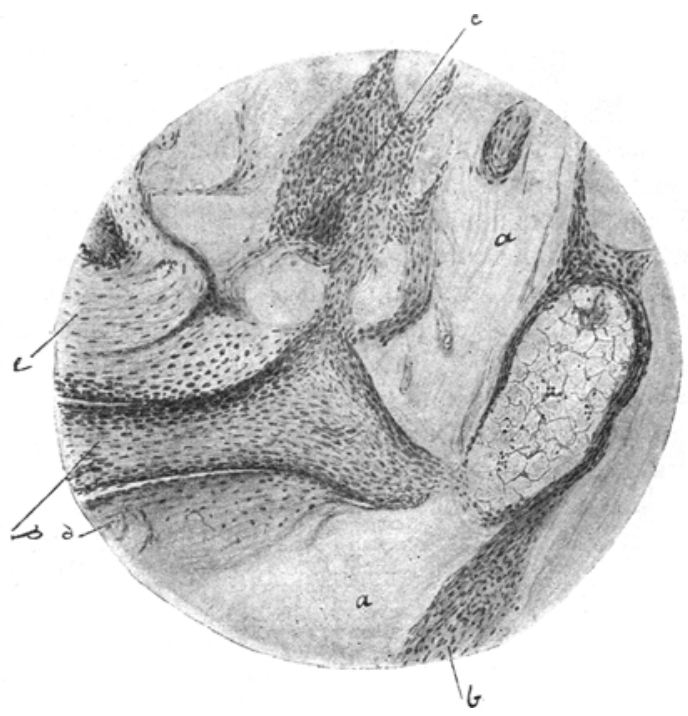

Fig . 9. Mitte der nekrotischen Zonen vollkommen abgegrenzte Knocheninseln und daneben Stränge, die von der Neuproduetion der Dura in die Diploëhöhlen gehen und von hier aus in die Dura.

An den Punkten, wo die Ossification sich noch nicht ganz vollzogen hat, sieht man gleicher Weise diese Stränge von Osteoblasten, die, mit den Gefässen vereinigt, von der Dura und dem Periost aus in die von früher her existirenden haversischen Canäle eindringen, um sich mit den osteoblastischen Productionen, die die Diploëhöhlen ausfullen, zu verbinden.

Besonders auffallend ist die reiche Vascularisation des

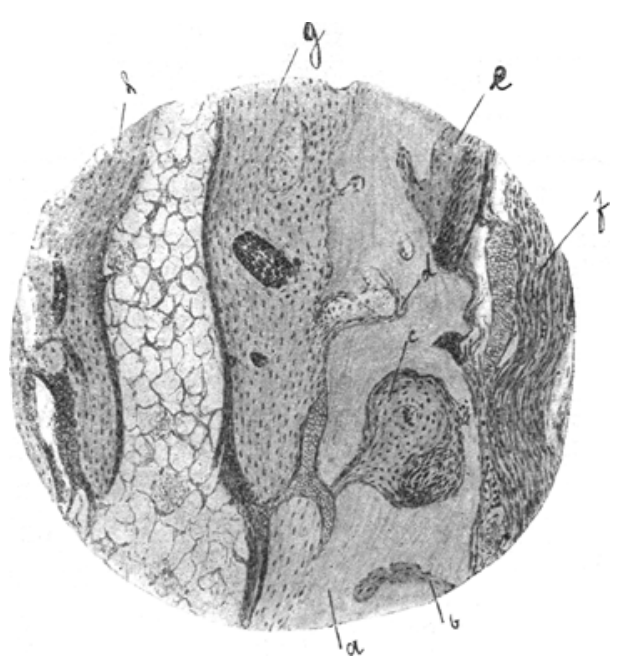

Fig. 10.

Knochens; man sieht denselben in allen Richtungen von weiten Canälen durchzogen, in deren Lumen die von Leukocyten umgebenen Gefässe ziehen. 
Mitten unter ihnen bemerkt man spärliche Bindegewebselemente an den Punkten, wo die Ossification noch nicht begonnen ist. Dies muss meiner Meinung nach als die erste Phase des Substitutionsprocesses betrachtet werden, eine Phase, in der die Absorption des Knochens und die Erweiterung der Canäle stattfindet, worauf dann die andauernde Phase der Ossification folgt.

In den Präparaten aus dieser Periode muss noch eine andere interessante Erscheinung hervorgehoben werden, nämlich das Fehlen des Ossificationsprocesses an der duralen und periostalen Oberfläche des nekrotischen Knochens. In der That sind hier nur Inseln neugeformten Knochens zu bemerken, verbunden mit Finbuchtangen und Dellen, in welchen sich die von der Dura und dem Periost herkommenden Elemente anhăufen und einnisten, als ob eine progressive Corrosion und Absorption des Knochens selbst stattfände, den ein osteogenetisches Gewebe zu ersetzen beginnt, welches an den von dem nekrotischen Theil am meisten entfernten Stellen Spuren von Ossification zeigt. Ferner bricht sich diese Production an den Punkten Bahn, wo sich haversische Canäle befinden, so dass die allgemeine Substitution schnell vor sich geht. Die Stelle, wo der Knochen unterbrochen wurde, ist fast völlig durch Balken neugeformten Knochens hergestellt, jedoch existirt noch eine zwischenliegende, ausschliesslich aus faserigem Bindegewebe bestehende Zone.

Fassen wir die aus dieser ganzen Reihe hervorgehenden Resultate zusammen, so finden wir, das der resecirte, von den weichen Theilen ganz befreite und reimplantirte Knochen beständig der Nekrose entgegengeht, einer totalen und vollständigen, von den ersten Momenten an zu constatirenden Nekrose. Dann beginnen die Corrosions- und Substitutionserscheinungen, die sowohl auf der Oberfläche der Dura, als auf der des Periosts, ebenso in den haversischen Canälen und den primitiven Diploëhöhlen vor sich gehen. In Folge dessen beginnt man im Innern des Knochens selbst zuerst Osteoblasteninseln und -Stränge zu sehen, je nachdem der Mikrotomschnitt transversal oder tangential auf die haversischen Canäle gefallen ist. In diese letzteren dringen die von der Dura und dem Periost kommenden Osteoblasten ein und erweitern sie, um sich darauf zu ossificiren. Dann hat man das charakteristische Bild der letzten Perioden vor sich, in denen man nur runde oder strangartig verlängerte Inseln und Streifen neuen Knochens sieht, inmitten der nekrotischen Masse, und von dieser durch eine scharfe Scheidelinie getrennt. Diese Substitution hört lauch in den spätesten Perioden nicht auf, noch vermindert sie sich, setzt sich vielmehr lebhaft fort, so dass, meiner Meinung nach, der !definitive Ausgang in der völligen Substitution besteht, einer Thatsache, durch die Barth's Ansichten bestätigt werden.

Charakteristisch ist es ferner, dass diese Substitution in den von uns beobachteten Perioden grösstentheils längs den alten haversischen 
Canälen und Diploëhöhlen stattfindet, wälırend sie auf der Oberfläche der Jura und des Periosts sehr gering ist; denn hier sieht man nur sehr kleine Knocheninseln, während vielmehr die Corrosions- und $\mathrm{Ab}$ sorptionserscheinungen vorwalten, die unter dem Einfluss eines neubildenden, an Gefässen und Elementen reichen Gewebes stehen. Diese letzteren nehmen in den tiefsten und von der nekrotischen Oberfläche am meisten entfernten Theilen das Geprïge von Osteoblasten an und gruppiren sich in ostëoidischen Balken.

Eine andere bemerkenswerthe Thatsache besteht darin, dass dieser Substitutionsprocess, so lebhaft er auch sein mag, hier weniger schnell und allgemein verläuft und sich langsamer entwickelt, als derjenige, den wir sich vollziehen sehen, wenn nur einzelne Knochensplitter am Periost hängen bleiben. Wir wollen hierbei nicht einmal in Rechnung ziehen, dass, während dort in gleich langen Perioden die Continuität des Knochens vollkommen wieder hergestellt war, dieselbe hier freilich wohl erreicht wird, aber nur scheinbar, da wir hier in Wahrheit, auch noch am Ende, zwischen den Resectionsrändern und dem reimplantirten Discus eine Zone faserigen Bindegewebes finden.

Lïsst man aber auch diese Thatsache bei Seite, obwohl sie jedenfalls als der definitiven Solidität des Schädeldaches nachtheilig betrachtet werden muss, so beunruhigen mich doch die schweren Folgen, die aus diesem grossen nekrotischen Knochenstück entspringen können. Es liegt hier lange inmitten der Gewebe, wie ein Caput mortuum, und stellt eine fortwährende Gefahr dar, bevor es ganz aufgezehrt und ersetzt ist. In dieser Besorgniss bestärkt mich noch die Häufigkeit später Eiterungen, die ich in Verlauf meiner Versuche bei Thieren, die vollständig per primam geheilt waren, habe auftreten sehen.

Besonders interessant ist ferner das Experiment von 2 Monaten, wo der Knochen fast ganz absorbirt ist, ohne dass sich irgend welche Reaction von Seiten des Periosts und der Dura hemerken lässt, ausgenommen auf dem Niveau der Ränder. Dieser Fall mag, es ist wahr, eine Ausnahme bilden, eine Ausnahme, die, wer weiss, welchen Coëfficienten zuzuschreiben ist. Jedenfalls dürfen wir bei der Abschätzung dieser Methode nicht vergessen, dass der Fall vorkommen kann, wo das reimplantirte Stück nicht durch Knochen, sondern einfach durch faseriges Bindegewebe ersetzt wird.

Periostmuskellappen ohne Reimplantation des resecirten Knochens.

Diese Experimente sind alle mit Kaninchen gemacht worden, mit weiten Defecten, die alle in der gewöhnlichen Weise mit der Scheere aus- 
geführt wurden. Die Basis betrug 2-21/2 cm, die IÖ̈he $1-12 \mathrm{~cm}$. Von den 5 operirten Thieren blieben 4 leben, von denen ich eines nach 3, ein anderes nach 4 Monaten tödtete. - Das Periost war beim Operationsact vollkommen erhalten geblieben. -

19 Tage. Junges Kaninchen. - Medianer Defect von $2 \mathrm{~cm}$ auf $1 \mathrm{~cm}$ Höhe mit der Scheere ausgeführt. Makroskopisch bemerkt man eine geringe Verdickung an den Rändern der Resection und eine dunne gespannte und glänzende Schicht Bindegewebe.

Bei der mikroskopischen Untersuchung nimmt man an den Knochenrändern Nekrose wahr,_die sich von den Sectionsoberflächen eine gute Strecke hindurch längs dem Knochen verbreitet. Die Dura und das Periost sind dicker geworden und reich an Elementen, von denen die am meisten centralen das Gepräge von Osteoblasten tragen und, in Balken angeordnet, schon einen ausgesprochenen Ossificationsprocess darstellen, so dass sowohl die Sectionsoberflächen, als die duralen und periostalen mit einer Zone jungen Knochens bedeckt sind, die vollkommen abgegrenzt und balkenförmig angeordnet ist. Von den Rändern aus werfen sich so. wohl Dura als Periost in den Defect und vereinigen sich, um eine einzige fibröse und stark vasculirte Schicht mit zahlreichen Zellen zu bilden, ohne irgend welche Spur von Ossification, nicht einmal einer beginnenden.

3 Monate. Junges Kaninchen. - Gewöhnlicher medianer Defect

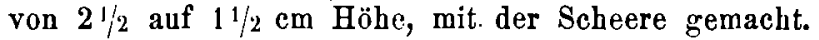

Der makroskopische Befund bietet nichts Interessantes.

Mikroskopisch hat man einen histologischen Befund, der sich vom vorhergehenden nicht wesentlich unterscheidet; der Knochen tritt therall als wiederhergestellt auf und die Ränder zeigen von nekrotischen Stellen keine Spur mehr; der Defect ist mit einem fibrösen sklerotischen Bindegewebe ausgefullt, zu dessen Bildung einerseits die Dura, andererseits das Periost beitragen.

3 Monate. Junges Kaninchen. In den Präparaten bemerkt man einerseits, dass der Knochenrand in allen seinen histologischen Details vollkommen im normalen Zustande erhalten ist; mit demselben steht ein skleröses, fibröses, durch das Zusammenfliessen von Dura und Periost gebildetes Bindegewebe in Zusammenhang, das eine der Höhe des durchschnittenen Knochens entsprechende Dicke besitzt, und das an der Stelle des Defects fortgenommene schädeldach ersetzt. Die einzige histologische Eigenthumlichkeit, der wir hier begegnen, ist die Gegenwart jungen Knochens, der sich an den früheren angelagert hat. Dieser hat in einigen Präparaten die Form eines Streifens, in anderen dagegen bietet er das Aussehen einer Insel oder eines Blockes dar, der unmittelbar in der Nähe des früheren Knochens liegt und mit demselben durch ein fibröses Bindegewebe verbunden ist. Und diese Neubildung hat hier noch nicht ihr Ende gefunden, wie man aus der Gegenwart einer beschränkten Osteoblastenzone ersieht, die den neugebildeten Knochen uberall umgiebt.

$4 \mathrm{M}$ on a te. Junges Kaninchen. Der mikroskopische Befund ist mit dem vorhergehenden fast identisch. 
Dieses Resultat war übrigens vorhergesehen in Anbetracht der klinischen Thatsachen. In der That hat man klinisch bei grossen Defecten nur ausnahmsweise den Fall gesehen, dass der Knochen sich mit dem periostalen Muskellappen allein wieder gebildet hätte. In der Literatur laabe ich nur 2 derartige Fälle erwähnt gefunden. Der eine ist der von Postempski, über den Seafi Bericht erstattet hat, der andere der von $\mathrm{Oechini.} \mathrm{In} \mathrm{beiden} \mathrm{Fällen} \mathrm{hat} \mathrm{die} \mathrm{Xeu-}$ bildung von Knochen stattgehabt, und zwar im ersten in Form von dünnen Lamellen, im zweiten in Form von Knochenblöcken. Mein Zweek bestand weniger darin, die an sich nicht in Zweifel zu ziehende Thatsache einer in unserem Fall nicht zu erwartenden Ossification festzustellen, als vielmehr, die Modalitäten derselben zu reproduciren und, wenn möglich, die Ursache aufzufinden. Nun bewegt mich der von mir erhaltene histologische Befund, der unzweifelhaft den Beweis geliefert hat, dass ein Ossificationsprocess stattgefunden hat und dass dieser (wenn auch mehr auf die Ränder der Continuitätsunterbrechung beschränkt) noch fortdauerte, anzunehmen, die ausgebliebene Ossification müsse dem Fehlen eines Stachels und eines Modells zugeschrieben werden.

Diese Factoren sind im gegenwärtigen Fall ausschliesslich auf die Peripherie des Defects beschränkt, wo wir gerade die Spuren einer Neuproduction von Knochen wahrzunehmen im Stande waren. Ich glaube daher, dass die Verwendung dieses Lappens, so werthvoll auch die äusserst schnelle Herstellung desselben sein mag, sehr eingeschränkt bleiben muss, nicht sowohl wegen der Gefahr eines Hirnbruchs (wie einige Autoren behauptet liaben), der nur von Suppurationsprocessen abhängt, als im Hinblick auf die Eventualität eines Traumas, das eine directe Wirkung auf das Gehirn ausüben könnte.

Osteoplastischer Lappen mit temporärer Resection des anden weichen Theilen hängen bleibenden Knochens in seiner ganzen Dicke.

In dieser Reihe von Versuchen wollte ich das Schicksal der resecirten Disken studiren in denjenigen Verfahren von temporärer Cranioresection durch die ganze Dicke des Schädels; deren Vorläufer Chal ot gewesen ist und die zuerst mit einigen Modificationen von Wagner in die Praxis übertragen sind. Diese Verfahren werden heutigen Tages in weitem Umfange angewandt wegen der in dieselben eingeführten nützlichen Modificationen und weil ein ganzer Vorrath von Instrumenten geschaffen worden ist, der ihre $\Lambda$ usführung wesentlich erleichtert. Es scheint uns daher der Mühe werth, uns mit denselben 
zu beschäftigen, um zu sehen, ob sie in Wahrheit so sebr den übrigen Verfahren überlegen sind, dass man ihnen den Vorzugr geben müsse.

Ich habe das Experiment mit 2 Hunden gemacht, aber in der That mit schlechten Resultaten. Mit einem feinen Hohlmeissel unterbrach ich den Knochen, indem ich demselben ungefähr die Form eines grossen_C gab; und nachdem seine ganze Dicke bis zur Dura unterbrochen war, zerbrach ich den noch intacten Theil des Knochens mit einem Levatorium; auf diese Weise wurde der am Periost anhangende Discus aufgehoben, um nachher wieder eingesetzt zu werder. Da nun aber bei den Hunden, deren Schädeldicke ziemlich beträchtlich ist, der Sectionsdefect, den ich nothwendig mit einem Hohlmeissel machen musste, sehr weit war, so konnte ich nicht verhindern, dass der Knochendiscus hinabsank. Ausserdem war die Fractur der noch intact gebliebenen, an der Basis des Iappens gelegenen Strecke des Knochens unregelmässig ausgefallen, so dass ich in einem Falle das Vorhandensein einer Spalte in der Richtung nach der Schädelbasis constatiren konnte; ferner wurde der Perioststiel bei der Abgrenzung des Knochenstiels, den ich nachher zerbrach, so misshandelt und reducirt, dass das Gelingen des Experiments compromittirt war. Zu allem diesem kommt die ausserordentlich lange Dauer des Operationsactes, folglich fast die Gewissheit, dass Sepsis eintreten würde, wie es bei den beiden Hunden geschah. So ist es leicht verständlich, dass ich mich entscbloss, Kaninchen als Versuchsthiere zu benutzen. Auch wollte ich nicht zum Trepan greifen, obwohl die Technik durch denselben bedeutend abgekürzt worden wäre, wegen der tiefen Alterationen, die derselbe im Knochen hervorruft, wovon ich mich in den vorhergehenden Versuchsreihen überzeugen konnte - Alterationen, die nafürlich bei der Wertbabschätzung des Verfahrens in's Gewicht gefallen wären. - Bei den Kaninchen hingegen, deren Parietalen sehr dünn sind, ist es möglich, mit einer kleinen scharfen, kurzen und feinen Scheere den Knochen zu durchbohren. Wenn man dann der Scheere eine Tangentenrichtung giebt, indem man eine Verletzung der Dura vermeidet, lässt sich der Lappen in der gewollten Weite und Form abgrenzen, ohne die weichen Theile zu misshandeln. Schliesslich liess ich eine sebr beschrünkte Knochenstrecke übrig, die ich darauf zerbrach, indem ich den Discus mit einer feinen Zange aufhob. So gelang es mir, 4 Kaninchen mit Erfolg zu operiren, bei einer Versuchsdauer, die sich von 5 Tagen bis auf $2 \frac{1 / 2}{2}$ Monate ausdehnte. Ich trug immer Sorge dafür, sehr weite Defecte im Verhältniss zu der Grösse des Thieres hervorzubringen, von $21 / 2-3 \mathrm{~cm}$. 
Experiment 1. Junges Kaninchen. Es wird links ein $21 / 2 \mathrm{em}$ langer und $1 \mathrm{~cm}$ hoher Defect ausgefuhrt, indem man die Continuität des Knochens auf drei Seiten unterbricht und ihn unten zerbricht, so dass man ihn am Periost und am Muskel anhängen lässt, welcher letztere eine Art von Scharnier bildet, vermittelst dessen sich der wieder in situ gebrachte Knochendiscus bewegen lässt. Dauer des Versuchs 5 Tage.

Makroskopisch ist nichts bemerkenswerth, was sich von dem bei einer Fractur in dieser Periode gewöhnlich Vorkommenden unterscheidet, abgesehen von einer geringen Blutung, die sich von dem Fracturpunkt uber die Dura verbreitet und die dem Knochen benachbarten parietalen und Muskelgewebe infiltrirt.

Mikroskopisch bemerkt man an den Rändern des Defects eine auf die Resectionsoberfläche beschränkte Nekrose, die sich aus dem Aussehen der Knochenzellen abnehmen lässt, die zum Theil verschrumpft, entstellt und verkleinert, zum Theil bleich, farblos wie Schatten und zu einer pulverigen Masse reducirt sind, die den Knochenkörper anfült. Von den haversisehen Canälen sind einige leer, andere voll von Blutgerinnsel. In den Diploëhöhlen sieht man weit ausgedehnte Hämorrhagien mit einer nicht unbedeutenden Infiltration. Auch hier sind die Gefässe theilweise thrombosirt, während in anderen die Circulation thätig bleibt; dagegen zeigt sich im Periost und in der Dura eine intensive Infiltration, die sich auch ther die parostalen Gewebe verbreitet und sich immer mehr ausspricht, je mehr man sich dem Fracturpunkt nähert, wo man zahlreiche Blutktgelchen bemerkt, sowie eine leukocytäre Infiltration, die so intensiv ist, dass sie an jenem Punkt die periostale und durale Structur verdeckt. Auf dem Niveau des resecirten Knochendiscus offenbart sich ausser dieser Infiltration der Dura und des Periosts eine intensive Infiltration der Diploëböhlen und eine sich nur auf einige haversische Canäle und Diploëgefässe erstreckende Thrombose, mit beschränkten Nekrosen des Knochens, während sich dieser im Allgemeinen völlig vital erhält und seine Circulation normal bleibt; und die Nekrose trifft theils die am meisten peripherischen Theile des Discus, die den Sectionspunkten entsprechen, theils centrale Theile, namentlich dort, wo die Knochengefässe der Thrombose ausgesetzt sind; man bemerkt eine ausgebreitete epidurale Hämorrhagie.

Experiment 2. Kleines Kaninchen. Fs wird, wie gewöhnlich, links operirt. Dauer des Experiments 9 Tage. Makroskopisch stellen sich die Knochen als normal dar: auf der Fracturlinie ist ein rosenfarbiges weiches Gewebe eingeschoben.

In den mikroskopischen Präparaten bemerkt man sofort eine Verminderung der leukocytären Infiltration, dagegen zeigt sich eine sehr starke Reaction von Seiten der dem Periost und der Dura eigenthumlichen Elemente und eine intensive Neubildung von Gefässen. Diese Neuproduction von Gefässen und Zellen ist fast ausschliesslich auf den Fracturpunkt beschränkt; sie verbreitet sich nur unbedeutend längs der Resectionsränder und ist auf dem Niveau des herausgenommenen Discus sehr wenig ausgesprochen. Sie wirft sich auf den Fracturpunkt, wo man Auslăufer junger Bindegewebselemente sieht, die mit den Gefässen in den zwischen den Sectionsrändern liegenden Ranm eindringen und grösstentheils das noch existirende Infiltrationsgebiet substituiren, wo man zahl- 
reiche Lymphocyten in Cariolysis antrifft. Die Diploëhöhlen und haversischen Canäle sind erweitert und varicös geworden. Dort, wo in diesen letzteren die Circulation sich vital erhält, siebt man rings um die Gefässe concentrische Schichten polyedrischer Elemente, die sich auch rings um die Diploëhöhlen finden, indem sie dieselben bedecken, während wir in andern haversischen Canäien von allem diesem nichts finden. Man sieht in diesen einige entstellte and verkleinerte Elemente, die Blöcke von Substanz umgeben, die bald hyalinisch-homogen, bald vielmehr opak und körnig ist, sich schwach färbt und das Licht stark bricht.

Experiment 3. Ausgewachsenes Kaninchen. Kranioektomie links, etwas medial, nach derselben Methode. Der Defect ist $3 \mathrm{~cm} \mathrm{lang,} 2 \mathrm{~cm}$ hoch. Dauer des Experiments 20 Tage.

Sowohl in dem resecirten Discus als an den Resectionsrändern bemerkt man identische Thatsachen, d. h. nekrotische Gebiete ohne Flemente, die mit völlig vitalen Gebieten abweclsseln; nur mit dem Unterschied, dass, während an den Resectionsrändern das nekrotische Gebiet auf das Niveau der Sectionsoberfläche beschränkt ist, im Knochendiscus die vitalen Punkte mit den nekrotischen abwechseln, welche letzteren verhältnissmässig spärlich sind und in keiner irgend welchen Ordnung auftreten. Periost und Dura befinden sich in lebhafter Reaction und weisen einen grossen Reichthum an jungen Elementen mit grossem, eiförmigem Kern auf, die sich rings um den Knochen anhäufen, und zwar in den entfernten Theilen ohne Ordnung, jemehr sie sich jedoch dem Knochen nähern, in ubereinander gelegten Schichten und Zonen angeordnet. Diese jungen Flemente werfen sich, sowohl von der Dura als vom Periost aus, auf die Sectionslinie, so dass diese sich uns ganz voll von jungem Bindegewebe darstellt, in welchem wir eine Tendenz zu einer Anordnung von osteoidischen Balken bemerken, die sich einerseits auf den fruheren Knochen, sowohl auf die Ränder wie auf den Discus stutzen, während sie sich andererseits mit dem den Defect anfüllenden Bindegewebe vereinigen. An diese Balken und an den frtheren Knochen sehen wir die Osteoblastenzeichen angelagert, und wenn man den Knochen in unmittelbarer Nähe des Resectionspunktes und auf dem Niveau des resecirien Discus betrachtet, so sieht man die haversischen Canäle und Diploëhöhlen weit über das Normale hinaus erweitert, unregelmässig und voll von theils spindelförmigen, theils eiförmigen, dicht aneinander gedrängten Elementen, die, zusammen mit den neugebildeten Gefässschlingen, sich mit der duralen und periostalen Neuproduction, sowie mit dem den Sectionsdefect anfüllenden Gewobe verbinden. Diese Elemente dringen durch den vitalen Knochen hindurch bis in Punkte, wo sich Gebiete befinden, die nicht mehr vital sind, und durchziehen anch diese nach allen Richtungen hin. Rings um einige der erwähnten Canäle und Höhlen nimmt man Knochengebilde mit vollkommen vitalen Zellen wahr, die, so eng sie sich auch an den nekrotischen Knochen anschliessen, bei genauer Betrachtung sich als durchaus verschieden von dem letzteren erweisen durch eine ganz klare, feine Demarcationslinie, während der Farbenton, den die Grundsubstanz an diesen Stellen annimmt, dunkler und weniger gleichmässig ist, so dass wegen dieser Eigenthümlichkeiten, sowie wegen der Beziehungen zu der Osteoblastenproduction im Innern der Canäle sich 
mit Sicherheit behaupten lässt, dass es sich bier um neugebildeten, den ehemaligen nekrotischen ersetzenden Knochen handelt.

Experiment 4. Grosses Kaninchen. Kranioektomie wie früher ausgeführt. Dauer des Fxperiments $21 / 2$ Mowate. Die Continuität des Schädels ist eine vollkommene, abgesehen davon, dass man auf dem Niveau des Punktes, der durch die Resection des Knochens getroffen wurde, noch eine feine Schicht Bindegewebe von fibrösem Aussehen bemerkt, das sich vom Periost nach der Dura hinzielut, während die Resectionsränder an Dicke zugenommen haben und der Knochen des Discus annähernd seine ursprtinglichen Dimensionen bewahrt hat.

Die Reaction von Seiten der Dura und des Periosts hat sich bedeutend vermindert, dagegen ist sie noch stark in den haversischen Canälen und den Diploëhöhlen, die sich uns unregelmässig und über das Normale hinaus erweitert darstellen; und zwar nehmen Erweiterung und Unregelmässigkeit dort zu, wo der resecirte Kuochen nicht mehr vital ist. An vielen Stellen bemerkt man, ausser einem grösseren Reichthum an Infiltrationselementen, die Erscheinung, dass der haversische Ossificationsprocess noch in Thätigkeit begriffen und Diploëböhlen sind mit Osteoblastenschichten bedeckt. Bei den am meisten peripherischen tritt die Knochenmetamorphose evident hervor. Grade diese sind es, die sich mit den Zonen neugebildeten Knochens verbinden, der vom nekrotischen Theil scharf unterschieden ist. An anderen Stellen ist die Ossification kaum angedeutet oder noch nicht einmal im Anfang begriffen.

Nimmt man Alles zusammen, so

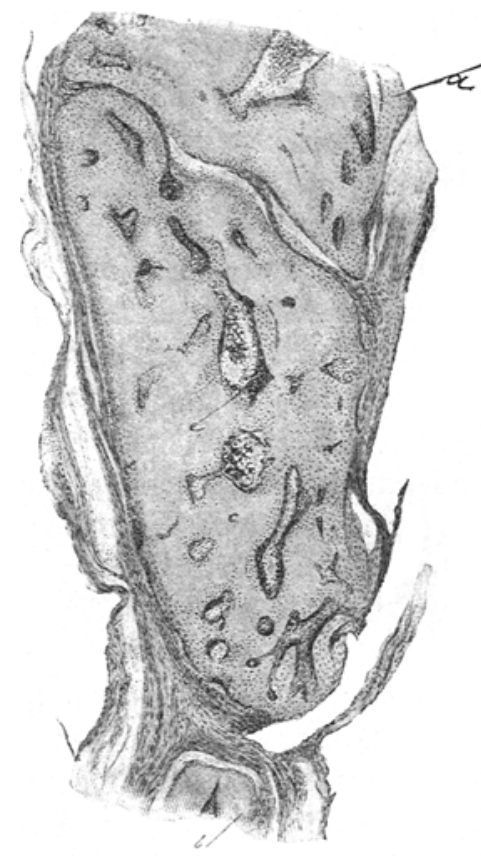

Fig. 11 . ergiebt sich, dass die Substitution der nekrotischen Gebiete noch vorschreitet, wenn auch weniger lebhaft, als in den vorhergehenden Perioden; und dieses gilt sowohl von dem Knochendiscus, als von den Sectionsrändern. - In beiden tritt ferner ein Streifen jungen Knochens klar hervor mit Elementen, die strang- oder balkenförmig oder concentrisch um ein Gefäss herum gruppirt sind. Dieser Streifen umgiebt die ganze Peripherie, sowobl auf der Seite der Dura, als auf der des Periosts, und unterscheidet sich durch seine tincturellen Figenschaften und das Aussehen der Zellen von dem benachbarten früheren Knochen, obwohl dieser noch vital ist. - An 
der Stelle, wo der Knochen unterbrochen wurde, befindet sich eine sehr geringe Strecke, die noch nicht durch Knochensubstanz wiederhergestellt ist. Man bemerkt hier ein faseriges, elementenreiches Bindegewebe, das von der Dura nach dem Periost geht und die Sectionsoberflächen mit einer Zone spindelförmiger Zellen von osteoblastischem Typus eng umschliesst.

Aus dieser Reihe von Experimenten geht klar hervor, dass, wenn man einen Knochendiscus in der Weise resecirt, dass er von den weichen Theilen, die ihn im normalen Zustande bedecken, nämlich von Periost und Dura nicht getrennt wird, ibn dagegen vermittelst eines hinlänglick grossen Stiels mit den weichen Theilen in Zusammenhang lässt, dieser Discus grösstentheils vital bleibt. Jedoch können beschränkte nekrotische Zonen vorkommen, atch unabhängig von der directen Wirkung des Traumas, nämlicb dort, wo die Gefässe der Thrombose verfallen sind. Jedoch werden diese Zonen von den ersten Perioden an durch einen von den alten haversischen Canälen ausgehenden Ossificationsprocess ersetzt. Diese im Anfang sehr thätige und lebhafte Ossification erscheint in den nachfolgenden Perioden langsamer, wenn auch immer in. Fortschritt begriffen. Die vollkommene Continuität zwischen dem resecirten Discus und den Rändern wird jedoch auch in diesen Perioden nicht erreicht, obwohl das Vorhandensein von progressiven Erscheinungen dem Gedanken Raum giebt, dass dieses Resultat später statthaben kann.

Dura und Periost mïssen also als zwei bedeutende Factoren des Reparationsprocesses der Schädeldefecte betrachtet werden. Aus unseren Versuchen geht jedoch nicht hervor, dass die Eine oder das Andere hierbei eine vorwiegende Wirkung ausübe. Ja, ihr osteogenetisches Vermögen scheint sich gleich zu stehen. Auch verhindert der Mangel eines der beiden Factoren, der Dura (die Einige als ein unentbehrliches Element betrachten), sicherlich nicht die Restitutio ad integrum. Die einzige Thatsache, die sich in diesen Fällen constatiren liess, war eine gewisse Verzögerung in den ersten Perioden. Aber dieser Erscheinung waren vielleicht andere Coëfficienten nicht fremd, wie die individuellen Eigenthümlichkeiten und der in den allerersten Perioden von Seiten des Gehirns ausgeübte Druck, welches ein wenig in der Richtung auf den Lappen hin emporragt. Uebrigens findet die Reparation statt und ist vollkommen, sei es, dass die Dura beibehalten oder fortgenommen wird, und zwar immer ohne intermediäre Knorpelbildung. Aber damit sich dieses osteogenetische Vermögen schnell und wirksam vollziehen 
kann, ist ein Stachel erforderlich. den in unserem Fall der frühere Knochen vertritt. Dies ist so gewiss, dass wir im Allgemeinen die Ossification nur dann erreicht haben, wenn entweder das ganze resecirte Stïck frei in situ belassen wurde oder Splitter desselben am Periost anhängen blieben. In diesem Punkt herrscht völlige Uebereinstimmung zwischen meinen Experimenten und denen Sacchi's und Pascale's, welche festgestellt haben, dass, wenn man den Knochendiscus fortnahm, die Reparation ausblieb und lediglich dic Bildung von faserigem Bindegewebe stattfand. Die Ansichten Berezowsky's stehen also in entschiedenem Widerspruch mit meinen Schlussfolgerungen, wie mit denen Sacchi's und Pascale's. Er berichtet in der That, dass er bei seinen Versuchen in Betreff des osteogenetischen Vermögens der Dura zu einer vollkommenen Reparation des Defects gelangt ist vermittelst neugebildeten Knochengewebes, welches ausschliesslich von der Dura ausging, während weder ich noch Sacchi oder Pascale den Defect je durch Knochengewebe dauernd wiederhergestellt gesehen haben, selbst wenn Dura und Periost belassen wurden. Es scheint mir, dass der Hauptgrund der Meinungsverschiedenheit in den Bedingungen, unter denen die Experimente ausgeführt wurden, zu suchen ist. Berezowsky hat nämlich einen Defect von nur $6 \mathrm{~mm}$ gemacht; wie soll man sich also darüber wundern, dass dieser reparirt worden sei vermittelst einfacher Neoformation, wenn auch von äusserst geringem Umfange, wie wir sie auch längs den Rändern beschrieben haben? In einem Punkte befinde ich mich jedoch durchaus in Uebereinstimmung mit Berezowsky, nämlich in Bezug auf das Schicksal der Knochensplitter. In der That hat auch er constatiren können, dass dieselben zu Grunde gegangen sind und dass ringsumher die Neoformation der Dura Platz gegriffen hat. Die ausschliessliche Aufgabe, die ich dem entweder theilweise oder ganz conservirten Knochen, der als Innest im Defect frei oder am Periost anhängend verbleibt, zuweisen muss, besteht gerade darin, dass er als Stachel, Modell und Leiter des Reparationsprocesses dienen soll. So hat auch meiner Meinung nach Barth vollkommen Recht, wenn er behauptet, dass bei den reimplantirten Bruchstücken und Disken die Nekrose die Regel bildet. Dagegen ist die Erklärung David's, so geistroll sie auch sein mag, nicht stichhaltig. Dieser spricht nämlich von einer verminderten Vitalität, in die der reimplantirte Knochen trete; diese sei eine vorübergehende, da in Wahrheit in den späteren Perioden Alles zum normalen Zustande zurückkehre: die Zellen sind bleich, schwer färbbar, der Kern mit Mühe zu unterscheiden, ja die Knochenkörperchen erscheinen leer, die haversischen Canäle wesentlich erweitert, 
die Diploëhöhlen sind infiltrirt und zeigen Spuren von einem körnigen Detrit, der intensiv färbbar ist. Dies sind die charakteristischen Eigenthümlichkeiten, aus denen David den Schluss zieht, dass es sich nur um eine Verminderung der Vitalität handele. Ich weiss hingegen nicht, was für andere charakteristischere Anzeichen die pathologisehe Anatomie bieten könnte, un den Beweis einer Nekrose zu liefern. Wahrscheinlich handelte es sich aber in David's Fall um Nekrose, da es unzweifelhaft ist, dass der Substitutions- und $\Lambda$ bsorptionsprocess bei einem Innest einen sehr verschiedenen Entwicklungsgang nehmen kann, wie auch Pascale bemerkt hat. So kann ein nekrotisches Knochenstück nach langer Zeit fast sein ursprüngliches Volumen mit nur geringen Spuren von Corrosion behalten haben, wie es in unserem Fall nach 30 Tagen vorgekommen ist, während ein anderes schnell aufgezehrt und im Wesentlichen sehr bald, wie in unserem anderen Fall von 25 Tagen, oder selbst vollständig, wie in unserem Fall von 2 Monaten, substituirt werden kann. Dies bemerke ich ausdrïcklich, weil es bei Experimenten mit verschiedenen Thieren sebr wohl vorgek ommen sein kann, dass der Experimentirende in der 6 . Woche wirklich einen Fall vor sich gehabt haben kann, in dem die Substitution vielleicht schon stattgehabt hatte, während derselbe in den vorhergehenden Perioden das entgegengesetzte Resultat gefunden baben könnte.

Valan ferner spricht von einer partiellen Nekrose, die nur an gewissen beschränkten Stellen eintrete, während sich andere vital erhalten sollen. Nun habe auch ich, wie Valan, in verschiedenen Präparaten einen ganzen Streifen lebendigen und vitalen Knochens beobachten können, der eine nekrotische Zone oder nekrotische, mit vitalen abwechselnde Inseln umgab. Jedoch konnte ich bei der weiteren Beschreibung der verschiedenen Präparate, in Lebereinstimmung mit Berezowsky, beweisen, dass diese Thatsache einer peripherischen Neuproduction rings um die Schicht zuzuschreiben sei, oder einer centralen medullären Neuproduction des Marks vermittelst von Osteoblasten, die sich in die Lücken und die ursprünglichen erweiterten haversischen Canäle einfiltrirt haben..

Hiermit will ich nicht völlig ausschliessen, dass ein Innest ausnahmsweise, namentlich in gewissen Dimensionen, gedeihen könne; aber, bevor das Gegentheil bewiesen ist, behaupte ich, dass wir dies in Zweifel ziehen müssen, und zwar umsomehr, als bei den Autoren, bei welchen vom Gedeiben gesprochen wird, abgesehen von wenigen Ausnahmen, die histologische Untersuchung vermisst wird, während man sich viel auf die makroskopischen charakteristischen Eigenthümlichkeiten verlässt, welche leicht täuschen können. 
$\mathrm{Ja}$, ich bin geneigt, den Zellenelementen eines resecirten und reimplantirten Discus noch entschiedener, als Pascale es gethan hat, die Vitabilität abzusprechen. Ich muss sogar gestehen, dass ich in meinen Experimenten nie die Gelegenheit gehabt habe, Flementen zu begegnen, die durch ihre charakteristischen Eigenthiimlichkeiten und ihre topographische Lage an eine Permanenz des Lebens denken liessen, und zwar von den ersten Perioden an. Was ferner das Wesentliche im Substitutionsprocesse betrifft, so werde ich nach meinen Befunden zu Schlussfolgerungen geführt, welche von denen Pascale's weit abweichen. In der That, von einem Monate nach dem Operationsact an finde ich, dass das Verschwinden des nekrotischen Knochens ausschliesslich an Absorptionserscheinungen mit allen Charakterzügen des lacunären Typus und der Gegenwart von Gigantenzellen gebunden ist. Eben denselben Process sebe ich nach 21/2 Monaten in einigen haversischen Canälen als einen permanenten; in anderen hat die Ossification entweder stattgehabt oder ist in Werden begriffen. Folglich muss ich diese als die erste Phase des Substitutionsprocesses betrachten, an deren Stelle dann die Periode der Neuproduction von Knochen tritt vermittelst der Osteoblasten, die granulationsweise in die haversischen Canäle und die erweiterten und von den Detriten befreiten Diploëhöhlen vordringen. Ich leugne keineswegs, dass in diesem Moment die erwähnten Osteoblasten, indem sie sich vervielfältigen, mit dazu beitragen können, die Höblen, die sie ausfüllen, noch mehr zu erweitern; ja, ich bin der Ansicht, dass das Verschwinden der nekrotischen Knochensubstanz und ihre gleichzeitige Ersetzung durcb neue Knochensubstanz, wie Pascale annimmt, allein in diesem Sinne $\mathbf{z u}$ verstehen ist.

Zieht man ferner die Progressivität dieses Processes bis zur vollkommenen Substitution (die von Barth angenommen, aber von Pas cal e geleugnet wird) in Betracht, so werde ich durch meine Experimente zu der Behauptung geführt, dass der Substitutionsprocess vom Periost und der Dura aus eingeleitet wird in Form von sich übereinander legenden Balken und Schichten, die sich schon in den ersten Perioden an dem nekrotischen Knochen anlagern. Jedoch findet dieser Process des sich übereinander Legens längs des ganzen nekrotischen Stückes nicht obne Unterbrechung statt, sondern vielmehr in Zonen und Inseln, die einen Theil der nekrotischen Knochensubstanz in Contact mit der vom Periost und der Dura ausgehenden Production jungen Bindegewebes lassen, durch welches dieselbe aufgezehrt und zernagt wird, wie es die zackigen und an Einbuchtungen reichen Ränder zeigen, während in den haversischen Canälen und den Diploëhöhlen die Ossi- 
fication statthat. Endlich in den letzten Perioden, die ich zu beobachten in der Lage war, sieht man viele von den Canälen und Höhlen des nekrotischen Knochens aranz mit junger Knochensubstanz angefüllt und rings um die Oberfliche des l'eriosts und der Dura Inseln gleichfalls neugebildeten Knochens, so dass es scheint, als ob die Substitution stillstehe. Wenn man aber auch in diesen Perioden seine Aufmerksamkeit auf das junge Bindegewebe richtet, das an der Dura und dem Periost seinen Ursprung hat und den Knochendiscus von allen Seiten umgiebt, so bemerkt man, dass, während sich dieses einerseits zwischen den neugebildeten Balken einnistet, um den nekrotischen Knochen an den Stellen, wo sich kein junger Knochen übereinander gelegt hat, zu erreichen und zu zernagen, andererseits in den entfernteren Theilen in der Gegend der fibrösen Zone, die Periost und Dura begrenzt, die Elemente dieses jungen Bindegewebes den Charakter von Osteoblasten annehmen und den Grund zu neuem Knochen legen.

Hieraus folgen drei Thatsachen: ein beträchtlicher Zuwachs an Dicke von Seiten des nekrotischen Knochendiscus, das Vorwiegen der Canalisation in der ihm angehörigen Grundsubstanz und die Substitution von Ilöhlen und Canälen durch Knochensysteme.

An dem Punkt, wo die Continuität des Knochens unterbrochen wurde, komnit der Reparationsprocess sehr frülızeitig zum Vorschein (hierin stimmen meine Versuche mit denen der andern Experimentatoren überein), jedoch ist es mir nie gelungen, in meinen Befunden eine vollständige Wiederherstellung zu sehen, dagegen fand ich stets eine zwischenliegende Zone von Bindegewebe, die in den weiter vorgeschrittenen Perioden ein fibröses Aussehen hatte.

Natïrlich muss man bei der Abschätzung der Experimente vielen Factoren Rechnung tragen, denen sich übrigens auch der klinische Fall nicht entziehen kann. Es ist nothwendig, den allgemeinen Zustand in Betracht zu ziehen, und einer der wichtigsten Umstände besteht darin, dass das osteogenetische Vermögen und die Activität der Reaction ausserordentlich versehieden sind zwischen dem einen Thier und dem andern. So kann bei einem Thier eine beschränkte und sehr scliwache Substitution stattfinden und der Knochendiscus noch nach langer Zeit fortexistiren, während bei anderen (wie es mir gerade bei dem Experiment von 2 Monaten vorgekommen ist) der Absorptionsprocess so energisch ist und so schnell verläuft, dass sich die Substitution kaum durch ein schwaches und theilweises Uebereinanderlegen an der schon fast ganz absorbirten Oberfläche des nekrotischen Knochens vollzieht.

Was jedenfalls aus meinen Experimenten hervorgeht, ist die Pro- 
gressivität der Phasen und die Thatsache, dass eine grosse Verschiedenheit zwischen den Anfangs- und den Schlussstadien herrscht. Wenn ferner die von mir beobachteten Modalitäten der Substitution eine lange Dauer voraussehen lassen, so geben sie doch keinem $Z_{w e i f e l}$ über den Verlauf des Processes bis zur vollständigen Reparation Raum.

Sehr verschieden sind hingegen die Erscheinungen, die man beobachtet, wenn man den Discus am Periost anhängen lässt, so dass er wie vermittelst eines Scharniers bewegt werden kann. In diesem Falle stimmen meine Resultate mit denen Pascale's vollkommen überein. Der Knochendiscus bleibt nämlich grösstentheils vital, während einige Zonen zu Grunde gehen; dies sind begrenzte Zonen, die meistens mit Thrombosen der sie benetzenden haversischen Gefässe verbunden sind. Der Absorptions- und Substitutionsprocess ist hier gleichzeitig oder rührt grösstentheils von den haversischen Canälen und den Diploëhöhlen her, in denen die Circulation unversehrt geblieben ist und die an die erwähnten Zonen angrenzen. Eins habe ich aber auch bier nie constatiren können, nämlich die vollständige Reparation der Strecke, an welcher der Knochen unterbrochen worden ist, so dass ich hier selbst in den am meisten vorgeschrittenen Perioden einen feinen Streifen Bindegewebe eingeschaltet finde, das den resecirten und vitalen Discus mit dem Rande des Defects verbindet.

Gehen wir von diesen lediglich theoretischen Schlüssen zur Praxis über, so stehen wir nicht an, die Methode der Reimplantation des resecirten Stückes zu verwerfen und noch viel mehr die ganze Kategorie der lebenden oder todten Heteroinneste. Diese Methode ist sicherlich der des periostalen Muskellappens nicht überlegen, ja sie bringt, ohne die Schnelligkeit der Ausfuhrung zu besitzen, Gefahren mit sich, denen die letztere nicht unterworfen ist, wie z. B. die Gefahr des Drucks, den das wieder eingesetzte Stück durch die unregelmässige atypische Production die sich auf demselben bildet, ausüben kann, ferner der secundären Infection, die immer zu befürchten ist in Anbetracht des langen Verbleibens des nekrotischen Knochendiscus. Ausserdem entspricht sie nicht mit Sicherheit dem Princip der Osteoplastik, nämlich von den ersten Tagen an dem Gehirn einen dauerhaften und starken Schutz zu gewähren, denn sie kann der völligen $\Lambda$ bsorption und der Substitution durch fibröses Bindegewebe entgegengehen, wie es Sacchi, Pascale und ich bemerkt haben. Dagegen ist der osteoplastische, nach der Methode Durante's gemachte Lappen immer vorzuziehen, oder die Lappen mit temporärer Resection des Knochens in toto, der an den weichen Theilen hängen bleibt. In diesem letzteren Fall wäre jedoch eine Technik zu wünschen, die so weit als möglich 
den Gebrauch des Trepans und der Säge ausschlösse, die tiefgehende Alterationen in der Constitution des Knochens hervorrufen können und dagegen weniger complicirte und leichter zu handhabende Instrumente in Anwendung brächte. Bei diesen miisste der Gesichtspunkt der einfachen Section durch Druck vorwalten vermittelst starker und scharfer Schneiden, die in entgegengesetzter Richtung (antagonistisch) wirken, wie bei den Craniotomen Roncali's und Iuvara's. Bis man jedoch ị praktischerer Weise als bisher nach diesem Grundsatz verfährt, ziehen wir den osteoplastischen Lappen Durante's vor, für den nur der einfache Meissel erforderlich ist und der uns stets vorzügliche Resultate geliefert hat.

\section{Literatur.}

Wagner, Die temporäre Resection des Schädeldaches. Centralblatt f. Chirurgie 1889. 1891.

Tois on, De la trép. du crâne. Congr. fr. de chir. 1891.

Chipault, Chir. opér. du syst. nerv.

Padula, Chir. cranica.

Broca et Maubrac, Chir. cérébrale.

Ollier, Traité expérimentale et clinique des os. 1867.

Derselbe, De l'ostéogénèse chirurgic. (Revue chirurgicale.)

Pa scale, Innesti ossei. 1898. - (Scritti angur. in omaggio del. Prof. Durante.)

Flourens, Gaz. méd. de Paris. 1859.

Bêrézowsky, La dure mère organe ossificateur. 1899.

Poulet (bei Broca).

Wedemeyer (bei Chipault).

Mac Ewen, Obs. sur la transplant. oss. Revue de chir. 1882.

Derselbe, Brit. med. Journ. 1886.

Derselbe, Lancet. 1588.

Gerster (bei Chipault).

Jaboulay (bei Scafi), Tecnica ed indicazioni delle res. cran. Lyon. méd. 1890.

King und Spitzka (bei Scafi).

Mossé, Acad. de méd. de Paris. 1893.

Derselbe, Rech. sur la greffe oss. Gaz. hebd. de Montpellier. 1888.

A damkiewicz, Ueber Knochentransplantation. Wien. med. Blätter. 1889.

Frank and Churk, A contribution to brain surgery. Americ. Journ. of med. sc. 1890 .

Wolff, Die Osteoplastik. Langenbeck's Archiv. Bd. 4.

Buscarlet, La greffe oss. Paris 1891.

Schmitt, Leber Osteoplastik. Langenbeck's Archiv. Bd. 45.

A. Barth, Histologische Untersuchungen über Knochenimplantation. Beitrag zur path. Anat. 1895.

David, Leber die histologischen Befunde nach Reimplantation. Archiv für klin. Chirurgie. 1896. 
Sacchi, Chir. cérébr. Compt. rend. intern. 1874.

Valerio, Contributo allo studio degli innesti cranici e della craniectomia.

Giorn. med. del R. Esercito. 1901.

Valan (bei Valerio).

Müller, Centralblatt für Chirurgie. 1890.

König, Centralblatt für Chirurgie. 1890.

Biagi, Dell' influenza nervosa sulla consolidaz. delle fratture. Scritti angur. in omaggio del Prof. Durante.

Scafi, Tecnica ed indicazioni delle resezioni craniche.

Occh ini, Della trapanazione delle ossa del cranio. Giorn. interuaz. delle scienze med. 1881.

Debreuil, Presse médicale. 1837.

Il eine, Gaz. méd. de Paris. 1836.

P. Flourens, Théorie expérimentale de la formation des os. 1847 .

Klencke, Physiol. der Entzündung nach Regeneration inorganischer Gewebe.

Q. Vignolo, Sul potere osteogenetico della dura madre. 1893. Monitore zoologico.

Ca miniti, La dura madre nei processi di ossificarcine. La clinica chirurgica. 1901.

\section{Erklärung der Abbildungen.}

Fir. 1. Osteoplastischer Lappen Durante's. Frau, nach 11 Jahren und 7 Monaten. Koristka, 3 Obj. a Osteoblasten, $b$ Knochenbalken, $c$ Bindegewebe zwischen den Balken, d Metamorphose des Bindegewebes im Knochen.

Hig. 2. Osteoplastischer Lappen Durante's (mit Dura). Hund, 18. Tag. Koristka, $3 \mathrm{Obj} . \quad a$ Nekrotische Lamelle, $b$ neugebildeter Knochen in Balkenform, $c$ ehemaliger Knochen (Resectionsrand), $d$ Periost, e Dura.

Fig. 3. Osteoplastischer Lappen Durante's (mit Dura). Hund, 2 Monate. Koristka, 3 Obj. $a$ Nekrotische Lamelle, $b$ neugebildeter Knochen durch Uebercinanderlegung, $c$ neugebildeter Knochen rings um die früheren haversischen Canäle.

Fig. 4. Osteoplastischer Lappen Durante's (mit Dura). Hund, 4 Monate. Koristka, 3 Obj. a Nekrotische Lamelle, von der Neoformation umgeben, $b$ neugebildeter Knochen durch Uebereinanderlegung.

Fig. 5. Osteoplastischer Lappen Durante's (ohne Dura). Hund, 25. Tag. Koristka, Obj. b. $a$ Resectionsrand, $b$ Periost, $c, e$ nekrotische Lamellen, $d, f$ Neoformation von Knochen.

Fig. 6. Resection des Knochens; Reimplantation des resecirten Knochens. Kaninchen, 9. Tag. Koristka, Obj. b. a Periost, $b$ reimplantirter nekrotischer Knochen mit Leberbleibseln von Knochenzellen, $c$ haversische, der Corrosion ausgesetzte Canäle, $d$ Dura.

Fig. 7. Ibid, Hund, 1 Monat. Koristka, Obj. b. a Iura, $b$ alter, der Corrosion verfallener Knochen, $c$ neugebildeter Knochen, $d$ Periost.

Fig. 8. Ibid., Hund, 2 Monate. Koristka, Obj. b. a Reimplantirter Knochen, $b$, $c$ neugebildeter Knochen, $d$ haversische Production. 
282 XI. BIAGI, Ceber die Reparationsprocesse der Schüdelknochen.

Fig. 9. Ibid., Kaninchen, 2'/2 Monate. Koristka, Obj. 5. a, a Peripherischer Theil des reimplantirten nekrotischen und der Corrosion ausgesetzten Discus, $b, c$ unregelmässig erweiterte haversische Canäle mit reicher Infiltration duraler Elemente, $d$ Ossification dieser duralen Elemente an den entfernteren Stellen, $e$, $e$ neugebildeter Knochen.

Fig. 10. Ibid., dasselbe Kaninchen, $2^{1} / 2$ Monate. Koristka, Obj. 5. a Reimplantirter, nekrotischer Knochen. $b, c, d$ haversische, mit neugebildetem Knochen angefüllte Canäle, $e$ theils ossificirte, theils in Ossification begriffene Insel (auf der Oberfläche der Dura gelegen), $f$ Dura, $g, h$ neugebildeter Knochen in einer Diploëhöhle.

Fig. 11. Osteoplastischer Lappen mit temporärer Resection des Knochens in seiner ganzen Dicke (an den weichen Theilen anhangend). Kaninchen, $21 / 2$ Monate. Koristka, Obj. b. $a, b$ Resectionsränder, $c$ nekrotische Gebiete, $d$ vitale Gebiete, e haversische Knochenbildung. 